\title{
Globular cluster ejection, infall, and the host dark matter halo of the Pegasus dwarf galaxy
}

\author{
Ryan Leaman, ${ }^{1 \star}$ Tomás Ruiz-Lara, ${ }^{2,3}$ Andrew A. Cole, ${ }^{4}$ Michael A. Beasley ${ }^{\circledR}, 2,3$ \\ Alina Boecker, ${ }^{1}$ Katja Fahrion, ${ }^{1,5}$ Paolo Bianchini ${ }^{\oplus}$, ${ }^{6,7}$ Jesus Falcón-Barroso, ${ }^{2,3}$ \\ Jeremy Webb, ${ }^{8}$ Alison Sills ${ }^{\oplus},{ }^{6}$ Alessandra Mastrobuono-Battisti ${ }^{\oplus},{ }^{1}$ Nadine Neumayer ${ }^{1}$ \\ and Anna C. Sippel ${ }^{1}$ \\ ${ }^{1}$ Max-Planck Institut für Astronomie, Königstuhl 17, D-69117 Heidelberg, Germany \\ ${ }^{2}$ Instituto de Astrofisica de Canarias, Via Lactea s/n, E-38205 La Laguna, Spain \\ ${ }^{3}$ Departamento de Astrofísica, Universidad de La Laguna, E-38206 La Laguna, Spain \\ ${ }^{4}$ School of Natural Sciences, University of Tasmania, Hobart, TAS 7001, Australia \\ ${ }^{5}$ European Southern Observatory, Karl-Schwarzschild-Str. 2, D-85741 Garching, Germany \\ ${ }^{6}$ Department of Physics and Astronomy, McMaster University, Hamilton, ON L8S 4M1, Canada \\ ${ }^{7}$ CNRS, Observatoire Astronomique de Strasbourg, Universite de Strasbourg, UMR 7550, F-67000 Strasbourg, France \\ ${ }^{8}$ Department of Astronomy and Astrophysics, University of Toronto, 50 George Street, Toronto, ON M5S 3H4, Canada
}

Accepted 2019 December 10. Received 2019 December 5; in original form 2019 February 4

\begin{abstract}
Recent photometric observations revealed a massive, extended $\left(M_{\mathrm{GC}} \gtrsim 10^{5} \mathrm{M}_{\odot} ; R_{\mathrm{h}} \sim 14 \mathrm{pc}\right)$ globular cluster $(\mathrm{GC})$ in the central region $\left(D_{3 \mathrm{D}} \lesssim 100 \mathrm{pc}\right)$ of the low-mass $\left(M_{*} \sim 5 \times 10^{6} \mathrm{M}_{\odot}\right)$ dwarf irregular galaxy Pegasus. This massive GC offers a unique opportunity to study star cluster inspiral as a mechanism for building up nuclear star clusters, and the dark matter (DM) density profile of the host galaxy. Here, we present spectroscopic observations indicating that the GC has a systemic velocity of $\Delta V=3 \pm 8 \mathrm{~km} \mathrm{~s}^{-1}$ relative to the host galaxy, and an old, metal-poor stellar population. We run a suite of orbital evolution models for a variety of host potentials (cored to cusped) and find that the GC's observed tidal radius (which is $\sim 3$ times larger than the local Jacobi radius), relaxation time, and relative velocity are consistent with it surviving inspiral from a distance of $D_{\text {gal }} \gtrsim 700 \mathrm{pc}$ (up to the maximum tested value of $D_{\text {gal }}=2000 \mathrm{pc}$ ). In successful trials, the GC arrives to the galaxy centre only within the last $\sim 1.4 \pm 1$ Gyr. Orbits that arrive in the centre and survive are possible in DM haloes of nearly all shapes, however to satisfy the GC's structural constraints a galaxy DM halo with mass $M_{\mathrm{DM}} \simeq 6 \pm 2 \times 10^{9} \mathrm{M}_{\odot}$, concentration $c \simeq 13.7 \pm 0.6$, and an inner slope to the DM density profile of $-0.9 \leq \gamma \leq-0.5$ is preferred. The gas densities necessary for its creation and survival suggest the GC could have formed initially near the dwarf's centre, but then was quickly relocated to the outskirts where the weaker tidal field permitted an increased size and relaxation time - with the latter preserving the former during subsequent orbital decay.
\end{abstract}

Key words: galaxies: evolution-galaxies: star formation-galaxies: stellar content.

\section{INTRODUCTION}

Dense globular clusters (GCs) are found in host galaxies spanning 6 orders of magnitude in stellar mass $\left(6 \lesssim \log _{10} M_{*} \lesssim 12\right.$; Forbes et al. 2018) making them a ubiquitous and informative tracer of the star formation (e.g. Maschberger \& Kroupa 2011) and accretion histories of their host galaxies. Their tight chemical co-evolution with the field stars of the host galaxy (e.g. Leaman 2012) implies

^E-mail: leaman@mpia.de that their ages and metallicities help us quantitatively understand what type of host they formed in (e.g. Leaman, VandenBerg \& Mendel 2013; Kruijssen et al. 2019; Massari, Koppelman \& Helmi 2019), and how that galaxy was assembled (e.g. Tonini 2013; Beasley et al. 2018; Choksi, Gnedin \& Li 2018) - even if the small-scale details of their formation are still far from known. Their intrinsic luminosity means that they also serve as useful dynamical tracers in distant galaxies, where the spectroscopic estimates of the kinematics of the galaxy's field stars is not observationally possible (e.g. Zhu et al. 2016; Beasley \& Trujillo 2016; Beasley et al. 2016). 
Star clusters residing in the very central potential of their host galaxies, nuclear star clusters (NSCs), are the most extreme example of dense stellar systems. Study of these particular objects is crucial for understanding how gas and star formation can proceed with (or precede) the formation of the central massive black holes observed in most galaxies (e.g. Antonini, Barausse \& Silk 2015).

The formation of NSCs is thought to occur through a combination of two complementary (and possibly concurrent; Guillard, Emsellem \& Renaud 2016) mechanisms: in situ star formation from a central gas disc and accretion of star clusters. Assembly of the NSC has been shown to occur in part due to sporadic in situ star formation from a central overdensity or disc of gas (Loose, Kruegel \& Tutukov 1982; Milosavljević 2004; Schinnerer et al. 2006, 2008). This mode can operate prior to, or after any inspiralling GCs have arrived in the central region of the host. Direct evidence for in situ gas accretion is evident from the spatial distribution of very young stars in the Milky Way's (MW) NSC (Levin \& Beloborodov 2003; Lu et al. 2009; Bartko et al. 2009; Genzel, Eisenhauer \& Gillessen 2010; Feldmeier-Krause et al. 2015), as well as in the nucleus of the Sagittarius dwarf galaxy (Alfaro-Cuello et al. 2019).

Dry merging of GCs as they inspiral to the centre of a galaxy requires the GCs to form near enough to the centre (or be accreted on favourable orbits from satellites), such that their orbital decay due to dynamical friction occurs within a Hubble time (e.g. Gnedin, Ostriker \& Tremaine 2014). In this scenario, the NSC stellar populations may be old and metal poor, characteristic of the dense GCs from which they are formed. Numerical simulations have shown that this mechanism can not only reproduce the structure of the NSC in the MW, but also the internal kinematics (e.g. the ratio of rotational to random motions; $V_{\text {rot }} / \sigma$ ) of the stellar component (Antonini et al. 2012; Perets \& Mastrobuono-Battisti 2014; Tsatsi et al. 2017).

However not all GCs present the same density and structural properties. Old GCs, the possible building blocks of galaxy nuclei, are observed to typically have $2 \mathrm{D}$ half-light radii of $R_{\mathrm{h}} \sim 4 \mathrm{pc}$, however there exists a long tail to the size distribution of star clusters. So called, 'extended' GCs, show radii up to a factor of 10 larger than typical GCs (Misgeld \& Hilker 2011). Extended GCs are observed in a variety of environments: the outer halo of M31 (Mackey et al. 2006; Collins et al. 2009) and the MW where they are thought to have been accreted with low-mass dwarf galaxies (e.g. Laevens et al. 2014; Weisz et al. 2016), the inner halo (15-30 kpc) of M31 (Huxor et al. 2005), the outer discs of nearby lenticular galaxies (Larsen \& Brodie 2000), around giant elliptical galaxies (Harris 2009; Webb et al. 2016), and in dwarf galaxies (Hwang et al. 2014).

Regardless of a GC's internal structure or mass, these objects serve as interesting probes of the host galaxy tidal field - especially in low-mass dwarf galaxies. The interest in GCs in low-mass systems stems partly from the large fraction of the total galaxy mass which they make up. This mass fraction in turn places constraints on the initial mass of the GC at formation, which is a crucial boundary condition for the study of multiple populations in GCs (e.g. Larsen, Strader \& Brodie 2012). Their presence in low-mass galaxies, which have not undergone significant environmental processing (e.g. merging with other galaxies and their GCs), means that systems with masses below $M_{*} \lesssim 10^{7}$ and their GCs can provide an unaltered window to the primordial efficiency of clustered star formation (ElBadry et al. 2019).

The very presence of GCs in low-mass galaxies means that the tidal field must have been conducive to their survival over a Hubble time. As the central regions of a galactic potential are typically the most tidally destructive, GC survival in low-mass haloes may be aided if they initially formed in the outskirts (or were ejected there soon after formation, cf. Kruijssen 2015) and then migrated by dynamical friction to the inner region during their lifetime. This process of orbital inspiral is sensitive to the shape of the total density profile of the host galaxy, with the relatively high-mass ratios between GCs and dwarf galaxy hosts meaning that this process is expected to be important in the survival of the GC (Orkney et al. 2019). For example, Petts, Read \& Gualandris (2016) showed under which conditions the host galaxy's density profile may lead to the dynamical friction processes stalling, while Meadows et al. (2020) further illustrated the similarity in dynamical friction time-scales in cored and cusped density profiles.

Another aspect to take into account are the particular dark matter (DM) distribution of the host galaxies. The DM haloes of lowmass dwarf galaxies are expected to show the largest deviations from $\Lambda$-cold dark matter $(\Lambda \mathrm{CDM})$ predictions of a cuspy NFW (Navarro, Frenk \& White 1997) profile due to baryonic feedback effects operating at high efficiency relative to the gravitational potential energy (e.g. Governato et al. 2010; Di Cintio et al. 2014; Bermejo-Climent et al. 2018). Observationally estimating the inner slope of the DM density profile from galaxy kinematic tracers is extremely challenging. Degeneracies with orbital anisotropy, stellar mass-to-light ratios and halo geometry make robust measurements of the core size difficult. GCs in these dwarfs therefore indirectly provide a limiting understanding of the galaxy density profile, as the joint migration and evaporative dissipation experienced by the GC depends on the background potential, and therefore the ages and positions of the GCs at present can be crudely leveraged to provide an understanding of the likely host galaxy DM structure (e.g. Leung et al. 2019).

Recently, the discovery of low-mass star clusters $\left(10^{2} \mathrm{M}_{\odot} \leq\right.$ $M_{*} \leq 10^{4} \mathrm{M}_{\odot}$ ) in (sometimes extremely diffuse) low-mass dwarf galaxies has brought added interest to this field of study. For example, Amorisco (2017) used numerical simulations to show that the presence of such low-mass fragile clusters necessitates almost completely cored DM haloes for their host galaxies. Regardless of whether these may be extreme examples, the study of GC orbital evolution provides a unique window for measuring the density profile of dwarf galaxies in systems that are otherwise inaccessible (Lora et al. 2013; Arca-Sedda \& Capuzzo-Dolcetta 2017).

It can be observationally difficult to use GC survival as a constraint on the host potential, as typically neither the GC's initial distance prior to infall is known, nor its current deprojected distance. Kinematic information can be used to understand the latter, but the low masses of dwarf galaxies means that extremely precise $(\delta V \leq$ $5 \mathrm{~km} \mathrm{~s}^{-1}$ ) relative velocities are needed to distinguish scenarios where the GC is at rest, or simply passing through the central region on an eccentric orbit (cf. Webb \& Vesperini 2018). The total potential of the host is subject to degeneracies in multiple descriptive parameters, which coupled with sometimes approximate prescriptions for tidal dissipation of the GCs, makes it challenging to quantify the host potential in all but the most extreme cases (Contenta et al. 2018). Even in those cases, additional information on the structure and age of the GC may help provide a more comprehensive evolutionary picture which can self-consistently explain how the GC acquired its structure, not just whether it could survive in a particular potential.

To provide the strongest constraints on the host potential, and better understand the environmental evolution of the GC, we would ideally want a low-mass dwarf (more likely to have higher DM fraction) with a high-mass GC (such that it would have survived for 
a Hubble time) with kinematic, stellar population, and 3D distance constraints on the star cluster relative to its host. Below we describe how many of these aspects are present in the newly characterized star cluster in the Pegasus dIrr galaxy.

\subsection{The Pegasus dIrr galaxy and its star cluster}

The Pegasus dwarf irregular galaxy (DDO 216) is a currently isolated member of the Local Group $\left(D_{\mathrm{MW}} \sim 1 \mathrm{Mpc} ; D_{\mathrm{M} 31} \sim\right.$ $500 \mathrm{kpc})$ with comparable stellar and gas mass $\left(M_{*}, M_{\mathrm{HI}}=\right.$ $6 \times 10^{6} \mathrm{M}_{\odot}$; McConnachie 2012). While the orbit of Pegasus is poorly constrained, its projected velocity and distance from its closest neighbour M31 suggest it is currently not bound, and thus unlikely to have had significant interactions with either of the massive spiral galaxies in the Local Group since $z \sim 1-2$ (i.e. the last 6-11 billion years). Resolved spectroscopic studies of the red giant branch (RGB) stars in Pegasus by Kirby et al. (2014) revealed a statistically significant (solid-body) rotation signature of $V_{\text {rot }} \sim$ $10 \mathrm{~km} \mathrm{~s}^{-1}$ and comparable random motions $\left(\sigma_{\mathrm{LOS}} \sim 12 \mathrm{~km} \mathrm{~s}^{-1}\right)$ with systemic velocity of $V_{\text {sys, RGB }}=-179 \pm 1.5 \mathrm{~km} \mathrm{~s}^{-1}$. Together these provide an estimate of the dynamical mass-to-light ratio within the half-light radius $\left(R_{\mathrm{e}}=695 \pm 37 \mathrm{pc}\right.$ ) of $M_{\mathrm{dyn}} / L=39 \pm 8$ (Kirby et al. 2014), suggesting even in the inner regions the galaxy is still dominated by a DM halo.

The HI gas in Pegasus shows a velocity gradient comparable to the stellar component, however the structural and kinematic asymmetry of the H I distribution led Young et al. (2003) to suggest that the $\mathrm{H}$ I velocity field was primarily the result of stellar feedback producing large-scale bulk gas motions, rather than intrinsic rotation of the cold neutral gas. McConnachie et al. (2007) similarly speculated that the disparate morphology of the stellar and gaseous components in Pegasus was the result of the latter being shaped by ram pressure as Pegasus interacted with a large-scale (intragroup) gaseous medium in the outskirts of the Local Group.

A recent Hubble Space Telescope (HST) photometric study of the stellar populations of Pegasus revealed the presence of a central massive star cluster in Pegasus, DDO 216-A1 (Cole et al. 2017; hereafter C17). Despite initial identification of the cluster candidate in early CCD imaging by Hoessel \& Mould (1982) and later notes by Gallagher et al. (1998), the object dropped out of literature compilations of Local Group star clusters for the next decades. The resolved star HST photometry allowed C17 to re-identify and confirm the object as a bona fide star cluster at the distance of Pegasus. Analysis of the colour-magnitude diagram (CMD) showed the age and metallicity of the cluster's stellar populations to be old $(\sim 12 \mathrm{Gyr})$ and metal poor $([\mathrm{Fe} / \mathrm{H}] \sim-1.7 \mathrm{dex})$. The radial variation of different populations suggested no young or intermediate component to the star cluster, unlike the field stars in Pegasus. Most importantly for this study, the identification and analysis of RR Lyrae variable stars in the star cluster and the dwarf galaxy provided a differential 3D distance estimate of the GC relative to the galaxy centre of $D_{3 \mathrm{D}} \lesssim 86 \mathrm{pc}$. Fits to the radial number density of old stars revealed structural properties which place the Pegasus star cluster in the regime of an extended star cluster (2D half-light radius $R_{\mathrm{h}}=13.5 \mathrm{pc}$, concentration $c \equiv \log \left(R_{\mathrm{tid}} / R_{\mathrm{c}}\right)=$ $1.24 \pm 0.39$, and tidal radius defined from King profile fits to the stellar number density profile $R_{\mathrm{tid}} \geq 75 \mathrm{pc}$ ). As we shall see, this observed tidal radius is larger by a factor of 3 than the expected theoretical Jacobii radius in this location of the galaxy.

The extended structure, large intrinsic mass $\left(M_{\mathrm{GC}} \sim 2 \times\right.$ $\left.10^{5} \mathrm{M}_{\odot}\right)$, and large fraction of its host galaxy mass $\left(M_{\mathrm{GC}} / M_{* \text {, gal }}\right.$ $\sim 0.05$ ) make the Pegasus star cluster a unique object of study in its own right. When combined with the precise differential age and distance estimates relative to its host galaxy, it becomes a truly special window into studying also the formation and survival efficiency of (extended) star clusters in low-mass dwarfs, and the host galaxy gravitational potential. Its high absolute mass and relative mass fraction with respect to Pegasus provides a different regime to understand the DM profile of the host - as typical dwarfs of this mass with less massive star clusters offer little flexibility in the allowable profiles (e.g. Amorisco 2017). The survival and migration of such massive star clusters is also key to the build-up of NSCs in dwarf galaxies, some of which may survive as the dense cores of their host galaxy during accretion to the MW (e.g. $\omega$ Cen; Herwig et al. 2012).

In this work, we add to the observational characterization of the Pegasus star cluster by presenting kinematic data from spectroscopy of the host galaxy and the star cluster (Sections 2 and 3), as well as numerical and analytic models for understanding the co-evolution of the star cluster and its host galaxy potential (Sections 4 and 5). A scenario for the formation and migration of the star cluster, discussions on its link to NSCs, and the host galaxy DM profile are presented in Section 6. We briefly summarize our findings in Section 7.

\section{OBSERVATIONS}

The data analysed in this work are part of a Directors Discretionary Time program (GTC05-16BDDT) aimed at obtaining an integrated spectrum for the GC in the Pegasus dIrr with the OSIRIS imager and spectrograph, ${ }^{1}$ mounted at the 10.4-m Gran Telescopio Canarias (GTC) in the Observatorio del Roque de los Muchachos, La Palma. The observations were carried out using the long-slit mode for OSIRIS (length of $7.4 \mathrm{arcmin}$ ) in combination with the R2000B grism ( $R \sim 2000$ ), a $2 \times 2$ binning, and a 0.6 arcsec slit, which yielded a spectral resolution of $\sim 2.26 \AA$ covering a wavelength range from 3950 to $5700 \AA$. The observations were split into two different Observing Blocks (OBs) that were executed on the 2016 November 21 and 24 during dark time. The slit was centred on the GC DDO 216-A1 (RA: 23:28:36.34, Dec: 14:44:25.0) and aligned with the parallactic angle, as shown in Fig. 1 for OB1. The two OBs had slightly different conditions: an airmass of $\sim 1.0$ and seeing of 1.2 and $1.4 \mathrm{arcsec}$ for the first and second nights, respectively. The observations consisted of a total of two exposures of $1850 \mathrm{~s}$ (first $\mathrm{OB}$ ) and two exposures of $1825 \mathrm{~s}$ (second $\mathrm{OB}$ ), allowing us to extract usable spectra for the GC as well as for the DDO 216 regions surrounding the GC (see Section 3).

Fig. 1 is a false-colour image created from HST images in the F475W (Sloan $g$ ) and F814W (Broad $I$ ) filters. The total integration times were 34 and $37 \mathrm{ks}$, respectively, allowing photometry of individual stars down to the oldest main-sequence turn-off. The observations are described in detail in C17.

\section{DATA ANALYSIS}

We reduced the observed data with an IDL/PYTHON-based reduction pipeline designed to deal with GTC/OSIRIS long-slit spectroscopic data. The OSIRIS detector is composed of two separate CCDs (2048x4096 pixels), however we placed the GC in a reference position in the western CCD. Typical reduction steps were performed on

\footnotetext{
${ }^{1}$ More information on the OSIRIS instrument can be found at http://www. gtc.iac.es/instruments/osiris/
} 




Figure 1. Slit orientation for our spectroscopic observations of DDO 216-A1 overlaid on the HST imaging of Cole et al. (2017). The GC is shown in the inset. The inner red circle and outer orange circle show the half-light radius and total extent of the cluster ( 3 and 15 arcsec, respectively).

the frames from this CCD, including bias subtraction, flat-fielding, wavelength calibration (typical relative errors below 2.5 per cent), and cosmic ray removal (using an IDL version of the L.A. Cosmic procedure, van Dokkum 2001). Despite the low number of sky lines affecting our wavelength range, a sky subtraction was done using the widely used Kelson sky subtraction algorithm (Kelson 2003). For this, we first define the sky pixels that are unaffected by any source of extraneous light (foreground and background objects or DDO 216 contamination) from which the sky spectrum was constructed. Next, this algorithm obtains a characteristic sky spectrum from those sky pixels based on the knowledge of the CCD distortions and the curvature of the spectral features. Finally, the four fully reduced, sky-subtracted frames corresponding to the four exposures are combined to obtain the final science frame to be analysed.

Three different spectra, spatially representative of the GC, and the eastern and western parts of DDO 216, were extracted. The extraction was based on the light profile along the slit obtained by collapsing the combined image along the spectral direction. The GC spectrum was constructed from an aperture of $\sim 45$ pixels including those pixels that are 50 per cent brighter than the median value along galaxy regions closest to the GC. As the two OBs were observed in different days and at different times and parallactic angle, we have decided to extract the spectra for the eastern and western parts of DDO 216 using just the data from the first OB (which had better seeing) in order to avoid mixing regions with potentially different stellar populations and kinematics. The spectra were extracted from apertures of 80 and 110 pixels towards the east and west of the GC, respectively. These apertures comprise pixels which are three times brighter than the median sky level. The final signal-to-noise ratio $(\mathrm{S} / \mathrm{N})$ per pixel of the extracted spectra are $\mathrm{S} / \mathrm{N} \sim 30,11$, and 19, for the GC, eastern, and western sections of the galaxy respectively. The spectrum from the eastern section of the galaxy is discarded from the following analysis because of its low $\mathrm{S} / \mathrm{N}$.

\subsection{Deriving the GC kinematic properties}

To recover the line-of-sight velocity distribution (LOSVD), we used three full spectral fitting and cross-correlation codes: ULYSS, PPXF, and FXCOR, together with different sets of stellar population 
synthesis models (using both BaSTI and Padova isochrones), based on empirical libraries of single stellar population (SSP) spectra created from the MILES stellar population synthesis models. The broad choice of parallel analysis techniques was motivated by an attempt to characterize the systematics in this methodology in a regime of low intrinsic velocity differences. We did the kinematic analysis as a blind exercise; the first author (who ran the orbital infall simulations in Section 5 which the velocities will be compared to), did not share the result of these simulations until after all independent velocity estimates from five of the co-authors were collected (TRL, MB, AB, KF, and JFB). Below we summarize the general methodology of the various codes used in these estimations.

\subsubsection{PPXF}

Here, we describe the analysis with PPXF (Cappellari \& Emsellem 2004), which is also used to constrain the kinematics in STECKMAP (STEllar Content and Kinematics via Maximum A Posteriori likelihood), and fits a combination of template spectra to the GC spectrum. For the PPXF template library, we used all available SSP models from the MILES (Vazdekis et al. 2015) libraries, ${ }^{2}$ with a bimodal initial mass function (IMF, and power-law index of $\Gamma=1.3$ ) and different choices of isochrones (BaSTI versus Padova) and different chemical enrichment $([\alpha / \mathrm{Fe}]=0.4$ or scaled solar). We fit only for velocity and velocity dispersion and exclude any higher moments. Prominent sky residuals and emission lines were masked out before fitting. All three spectra were fit for kinematics with an additive polynomial which converged at degree 10 .

To determine conservative errors on the extracted best-fitting kinematics, we performed a Monte Carlo simulation of the velocity determination in two ways. In the first method, we randomly reshuffled the residuals of the best fit of the GC spectrum and added them to the best-fitting template spectra. Any residuals from masked areas were replaced by random values between the standard deviation of the residuals. We then let PPXF recover the LOSVD again. This process was repeated 100 times and the standard deviation of the recorded velocity and velocity dispersion were taken as our errors. The second method used a similar approach, but with the perturbations taken from Poisson draws of the observed flux in a given pixel of the spectrum. These uncertainty characterizations were performed for both spectra from the West of the galaxy, and the GC spectrum (OB1 was used for both cases). The final random uncertainty was taken as the standard deviation of the distribution of the 100 velocity estimates. An example of the PPXF fitted GC and galaxy spectra are shown in Fig. 2.

\subsubsection{ULYSS}

The kinematic measurements of the LOS velocity were also done with the spectral fitting code ULYSS (Koleva et al. 2009) for the GC and Pegasus West spectra. ULYSS performs a pixel-by-pixel fit to an observed spectrum by using a model constructed from a linear combinations of non-linear components. In this case, we used the MILES base-models (Vazdekis et al. 2010), which predict composite stellar population spectra as a function of age and metallicity. We used SSP models with a Kroupa IMF and performed fits within the wavelength range 4000-5500 A. Velocity

\footnotetext{
${ }^{2}$ The MILES models are publicly available at http://miles.iac.es and are based on the MILES empirical library of stellar spectra (Sánchez-Blázquez et al. 2006; Falcón-Barroso et al. 2011)
}
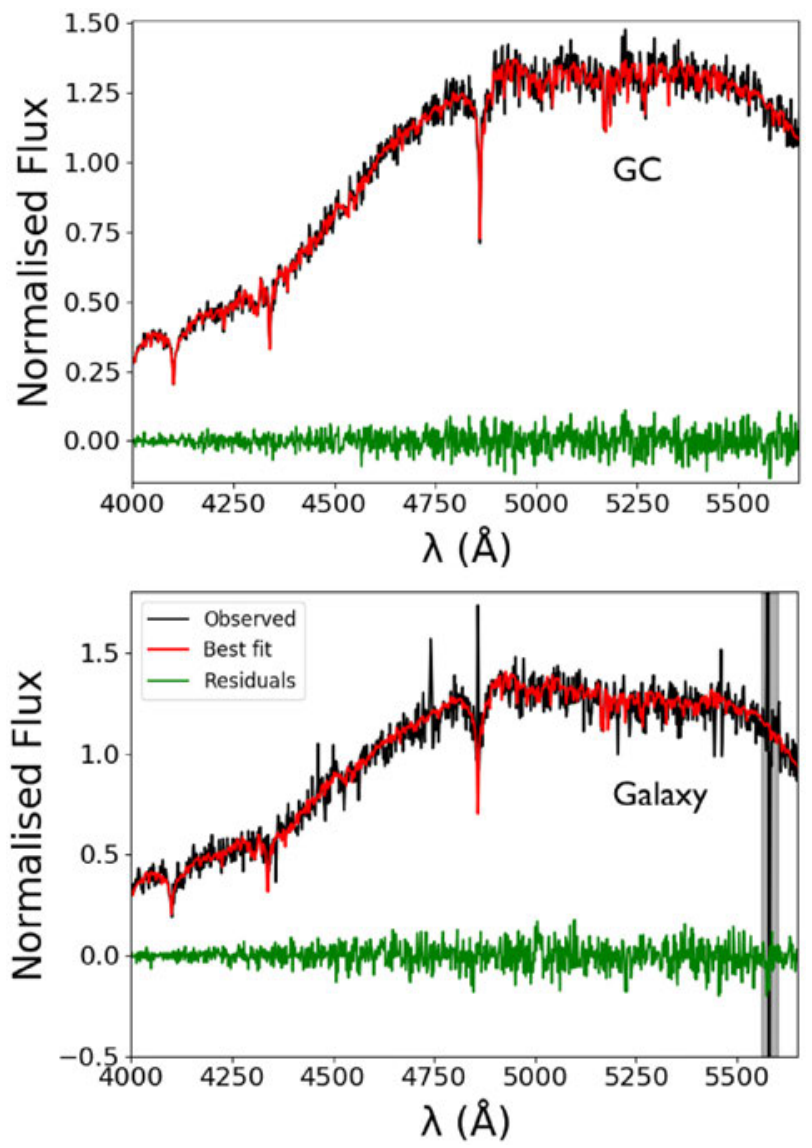

Figure 2. STECKMAP fits (red) to the GC spectrum (top) and galaxy spectrum (bottom). Observed spectrum is in black, residuals in green, and masked sky/emission lines in grey.

uncertainties were calculated via Monte Carlo simulations, where noise corresponding to $\mathrm{S} / \mathrm{N}=30 \AA^{-1}$ was added to the observed spectrum and the velocity was remeasured. The final velocity uncertainty was taken to be the standard deviation of the returned independent velocities.

\subsubsection{FXCOR}

We also directly cross-correlated the Pegasus West spectrum with that of the GC, using the IRAF package FXCOR. We ran the crosscorrelation on $\Delta \lambda=250 \AA$ wide windows, centred on seven different central wavelengths $\left(\lambda_{\mathrm{c}} \simeq 4150,4350,4620\right.$, 4850, 5100, 5350 , and $5620 \AA$ ). The relative velocity and internal error in resolving the cross-correlation peak returned by FXCOR as well as the other methods are discussed in Section 5.2.

\subsection{Stellar population modelling}

The stellar population properties were similarly derived using two different spectral fitting codes (PPXF and STECKMAP) as well as a line index-based measurement. Below we describe the general procedure used in each method.

\subsubsection{STECKMAP}

We assessed the stellar content of the GC with an alternative approach, which makes use of GANDALF (Gas AND Absorption 
Line Fitting, Sarzi et al. 2006; Falcón-Barroso et al. 2006) in order to remove the possible contamination from gaseous emission and STECKMAP (Ocvirk et al. 2006a, b) to properly recover the stellar composition of the analysed objects. This methodology has been extensively used and tested (e.g. Sánchez-Blázquez et al. 2014; Ruiz-Lara et al. 2015, 2016, 2017; Pérez et al. 2017), however a short description is provided here.

After the simultaneous fit of the stellar continuum and the emission lines (treated as additional Gaussians) is done by GANDALF, a pure absorption spectrum was obtained by subtracting the detected emission from the observed spectrum. Some small gaseous emission line contribution was found in $\mathrm{H} \beta(\mathrm{S} / \mathrm{N} \sim 4)$ on the $\mathrm{GC}$ spectrum, and more prominent $(\mathrm{S} / \mathrm{N} \gtrsim 10) \mathrm{H} \beta$ emission was detected in the galaxy spectrum. STECKMAP is able to simultaneously recover the stellar content and kinematics using a Bayesian method via a maximum a posteriori algorithm. However, we have decided to fix the stellar kinematics to those values found from PPXF (see Section 3.1) in order to avoid the well-reported metallicity and velocity dispersion degeneracy (Sánchez-Blázquez et al. 2011). The STECKMAP solution is regularized via a penalization function. This penalization means that smooth shapes for the stellar age distribution or age-metallicity relation (AMR) are preferred over highly oscillatory or patchy ones. The degree of smoothness is determined by the user by means of the smoothing parameters ( $\mu_{\mathrm{x}}$ for the ages and $\mu_{\mathrm{Z}}$ for the metallicites). In this case, we use $\mu_{\mathrm{x}}=100$ and $\mu_{\mathrm{Z}}=0.1$, although this choice might slightly affect the shape of the recovered SFH (star formation history), the general results are not modified. For detailed studies and discussions on how these two parameters might affect the recovered SFHs, we refer the reader to Ruiz-Lara et al. (2015, 2018b).

\subsubsection{Line index measurements}

As an alternative stellar population analysis we also used a method detailed in Martín-Navarro et al. (2018), which is a Markov Chain Monte Carlo (MCMC) method based on adaptively masking contaminated regions of the spectra while measuring line indices with pixel-by-pixel integration. We used the Cervantes \& Vazdekis (2009) definition of the $H \beta_{0}$ index, combined with the Fe5015, Fe5335 (Worthey et al. 1994), and Fe5270, Mgb, $H \gamma_{\mathrm{f}}, H \delta_{\mathrm{f}}$ standard line indices (Burstein et al. 1984). The combined line indices were compared with similar adaptive pixel integration of MILES SSP models (BaSTI isochrones) in order to compute the posterior likelihood of the light-weighted age, metallicity, and $[\alpha / \mathrm{Fe}]$. In this step, the models were convolved with a Gaussian kernel according to $\mathrm{FHWM}_{\mathrm{kernel}}^{2}=\mathrm{FHWM}_{\mathrm{GC}}^{2}+\mathrm{FHWM}_{\mathrm{LSF}}^{2}-\mathrm{FHWM}_{\mathrm{MILES}}^{2}$, where the line spread function $\mathrm{FHWM}_{\mathrm{LSF}}=2.1963 \AA$, and $\mathrm{FHWM}_{\text {MILES }}^{2}=$ $2.51 \AA$ (Falcón-Barroso et al. 2011), and the measured velocity dispersion of the GC is used to compute the $\mathrm{FWHM}_{\mathrm{GC}}$. SSP models constructed from both solar-scaled and $\alpha$-enhanced BaSTI isochrones (Vazdekis et al. 2015) were used in this MCMC method, and the priors on age, metallicity and $[\alpha / \mathrm{Fe}]$ were uniform within the ranges of the SSP model grid. The final posterior distributions and the median and 16th and 84th percentiles are discussed in Section 5.3.

\section{OBSERVATIONAL RESULTS}

\subsection{Observed relative velocity of the GC}

While there exist literature estimates of the systemic velocity of the RGB stars in Pegasus $\left(V_{\text {sys, RGB }}=-179 \pm 1.5 \mathrm{~km} \mathrm{~s}^{-1}\right.$; Kirby et al.
Table 1. Measured GC and galaxy velocities from each of five co-authours as described in Section 3.

\begin{tabular}{llccc}
\hline$V_{\mathrm{GC}}$ & \multicolumn{1}{c}{$V_{\mathrm{Gal}}$} & $\Delta V$ & Method & {$\left[\lambda_{\min }, \lambda_{\max }\right]$} \\
\hline$-142 \pm 4$ & $-155 \pm 11$ & $13 \pm 12$ & PPXF & {$[4760,5558]$} \\
$-161 \pm 1$ & $-175 \pm 2$ & $14 \pm 2$ & ULYSS & {$[4000,5500]$} \\
$-163 \pm 5$ & $-160 \pm 8$ & $-3 \pm 10$ & STECKMAP & {$[3900,5700]$} \\
$-159 \pm 3$ & $-153 \pm 4$ & $-6 \pm 5$ & PPXF & {$[3980,5690]$} \\
$-149 \pm 7$ & $-146 \pm 15$ & $-3 \pm 16$ & PPXF & {$[3900,5700]$} \\
$-152 \pm 4$ & $-155 \pm 9$ & $3 \pm 10$ & STECKMAP & {$[4760,5558]$} \\
\hline
\end{tabular}

Notes: Average: $\Delta V=3.0 \pm 3.7$ (ran.) \pm 8.7 (sys.)

Weighted average: $\Delta V=10.4 \pm 1.8$ (ran.) \pm 7.3 (sys.)

2014), ionized gas ( $V_{\text {sys, } \mathrm{H} \beta}=-172 \mathrm{~km} \mathrm{~s}^{-1}$; this work), and HI gas $\left(V_{\text {sys, HI }}=-183.3 \mathrm{~km} \mathrm{~s}^{-1}\right.$; Young et al. 2003), the presence of intrinsic rotation or peculiar motions in this low-mass dwarf makes comparison to the GC velocity intrinsically difficult. Therefore given the expected orbital velocities $\left(\leq 20 \mathrm{~km} \mathrm{~s}^{-1}\right)$ for Pegasus, velocity precision is maximized by analysing differential stellar kinematics of the cluster and host from the same spectroscopic observations. Below we present the analysis of the kinematic results derived from our long-slit spectroscopic observations of the GC and the Pegasus galaxy.

\subsubsection{Systematic uncertainties in velocity determination}

The blind experiment to determine the LOS velocity of the GC and the host galaxy, conducted by five of the co-authors, is summarized in Table 1. This exercise has revealed systematics of the order $\sim 15 \mathrm{~km} \mathrm{~s}^{-1}$ that may be inherent to many of these full spectral fitting codes (which were designed for use on high-mass galaxies with characteristic rotation and velocity fields of $V, \sigma \gtrsim 150 \mathrm{~km} \mathrm{~s}^{-1}$ ). While recent updates to PPXF (Cappellari 2017) include an analytic oversampling of the LOSVD designed to improve systematics in systems with $\sigma \leq \sigma_{\text {instrumental }}$, our tests all used these updated versions of the code and show that for low S/N spectra $(\sim 20$ $30 \AA^{-1}$ ) a scatter in recovered $V$ at these levels is difficult to avoid in that and other full spectral fitting codes.

As illustrated in Table 1, the random uncertainties in the relative velocity $\left(\Delta V=V_{\mathrm{GC}}-V_{\text {galaxy }}\right)$, which were estimated by Monte Carlo simulations with each of the codes, all return individual uncertainty estimates that are smaller $\left(\sim 10 \mathrm{~km} \mathrm{~s}^{-1}\right)$ than the range $\left(25 \mathrm{~km} \mathrm{~s}^{-1}\right)$ of velocities found from each of the six independent trials.

The absolute values of the velocity of any object, differ by $\sim 30 \mathrm{~km} \mathrm{~s}^{-1}$ across the trials - a factor of $\sim 10$ higher than the random uncertainty on $V_{\mathrm{GC}}\left(\sim 4 \mathrm{~km} \mathrm{~s}^{-1}\right)$ returned by the Monte Carlo noise injection simulations which produced the random uncertainty estimates. These discrepancies suggest, while our $\mathrm{S} / \mathrm{N}$ is moderate, that the results are not simply a manifestation of the data quality.

Some of these systematic variations can be traced to specific choices in the kinematic analysis. For example, systematic offsets appear when considering different wavelength ranges - a shift in the bluest wavelength (while keeping the total spectral range fixed to $600 \AA$ ) from 4400 , to 4800 , resulted in velocity shifts of 19 and $17 \mathrm{~km} \mathrm{~s}^{-1}$ for the GC and galaxy spectrum, respectively. To confirm this shift was not due to an error in wavelength calibration, we repeated the same exercise with galaxy spectra from the SAURON and CALIFA galaxy integral field unit (IFU) surveys and found a comparable change in LOS velocity of $15 \mathrm{~km} \mathrm{~s}^{-1}$ over the same set of changes to the wavelength range. 
Other choices reassuringly provide consistent results - for example the use of PYTHON versus IDL versions of PPXF resulted in no change to the returned velocity of the higher S/N GC spectrum, but a change of $-8 \mathrm{~km} \mathrm{~s}^{-1}$ to the lower $\mathrm{S} / \mathrm{N}$ galaxy spectrum. An additional test comparing the use of MILES versus E-MILES and BaSTI versus Padova isochrone-based models showed a mild $2 \mathrm{~km} \mathrm{~s}^{-1}$ offset for the former libraries occurred for both the GC and galaxy spectrum - but the differential velocity between the GC and spectrum was the same.

Clearly care needs to be exercised in our particular regime, as even if a well-resolved cross-correlation peak is localized, the systematic uncertainty on its zero-point seems to be a significant fraction of the instrumental resolution when all effects are accounted for. More study is needed to see how these effects may or may not change as a function of spectral resolution and S/N.

Returning to the purposes of our astrophysical investigation, Table 1 presents the ensemble LOS velocities measured for the GC and galaxy spectrum. In the case that all our errors are estimated consistently, a weighted average of the different $\Delta V$ values would be appropriate. However, if they are estimated differently, then a straight average is more statistically appropriate. We believe the latter to be true based on the extensive systematics described above, as well as the fact that all the returned internal uncertainties are smaller than the scatter in the trials - suggesting that the error budget is not fully, nor consistently captured across the different measurements.

The bottom of Table 1 shows the final relative velocity between the GC and the galaxy, $\Delta V$. In the case of the unweighted estimate, the random error is taken as the standard error on the mean, and the systematic error as the standard deviation of all estimates of $\Delta V$. In what follows, we use this straight mean on $\Delta V$ and the systematic error $\left(\sigma_{\Delta \mathrm{V}}\right)$ as it is the larger of the two $),{ }^{3}$ as our final value of the relative difference in velocity of the $\mathrm{GC}$ with respect to the centre of Pegasus: $\Delta V=3.0 \pm 8.7 \mathrm{~km} \mathrm{~s}^{-1}$.

For completeness, we also quote the weighted average $\left(\overline{\Delta V}_{\mathrm{w}}\right)$, with its random $\left(\delta_{\Delta \mathrm{V}, \mathrm{w}}\right)$ and systematic $\left(\sigma_{\Delta \mathrm{V}, \mathrm{w}}\right)$ uncertainties computed as:

$\overline{\Delta V}_{\mathrm{w}}=\frac{\sum\left(\Delta V_{\mathrm{i}} \sigma_{\Delta \mathrm{V}_{\mathrm{i}}}^{-2}\right)}{\sum\left(\sigma_{\Delta \mathrm{V}_{\mathrm{i}}}^{-2}\right)}$

$\delta_{\Delta \mathrm{V}, \mathrm{w}}=\sqrt{\frac{1}{\sum \sigma_{\Delta \mathrm{V}_{\mathrm{i}}}^{-2}}}$

$\sigma_{\Delta \mathrm{V}, \mathrm{w}}=\left(\frac{\sum \sigma_{\Delta \mathrm{V}_{\mathrm{i}}}^{-2}\left(\Delta V_{\mathrm{i}}-\overline{\Delta V}_{\mathrm{w}}\right)^{2}}{\sum \sigma_{\Delta \mathrm{V}_{\mathrm{i}}}^{-2}}\right)^{1 / 2}$

As a final note, our exercise of directly cross-correlating the galaxy and GC spectra with FXCOR (Section 3.1.3) found comparable results to the ensemble of full spectral fitting results: $\Delta V=$ $3.0 \pm 2.1$ (ran.) \pm 7.9 (sys.) $\mathrm{km} \mathrm{s}^{-1}$.

\subsection{Stellar populations of the star cluster}

The right-hand panel of Fig. 3 shows the line index-based measurements of total metallicity $([\mathrm{M} / \mathrm{H}]),[\alpha / \mathrm{Fe}]$, and age for the GC aperture, using the methodology described in Section 3.2.3. The split age distribution from the index measurements suggests that

\footnotetext{
${ }^{3}$ Quadrature addition of the systematic and random errors is not appropriate here as they are both non-Gaussian and correlated - hence we present them independently.
}

multiple stellar population components of the GC and host galaxy are detected in the central spectrum of the slit. The oldest component is in excellent agreement with the CMD-based age of $12.3 \pm 0.8 \mathrm{Gyr}$ found by $\mathrm{C} 17$. The index measurements show an alpha-enhanced stellar population of $[\alpha / \mathrm{Fe}]=0.35_{-0.07}^{+0.04}$, while the total metallicity, $[\mathrm{M} / \mathrm{H}]=-1.79 \pm 0.04 \mathrm{dex}$ is also consistent with the CMD fitting of $\mathrm{C} 17$, which found $[\mathrm{M} / \mathrm{H}]=-1.6 \pm 0.2 \mathrm{dex}$.

The normalized star formation rate (SFR) and AMR recovered for the GC and the galaxy from STECKMAP full spectral fitting are shown in Fig. 3 (black lines) using the MILES-Padova models. Error bars are computed as the standard deviation of the solutions of 25 Monte Carlo simulations by adding random noise to the best solution of the first realization and running STECKMAP with the same input parameters (see Section 3.2.2). In order to take into account the possible effect of such particular choice of parameters, following Ruiz-Lara et al. (2018a,b), we sample the parameter space using different set of parameters within reasonable ranges. The shaded area of Fig. 3 encompasses all such solutions, highlighting the robustness of the solution and the little dependence upon this choice.

The STECKMAP analysis of the GC spectrum shows a broad range of ages; with signatures of intermediate and young stellar populations at $\sim 4$ and $\sim 2$ Gyr respectively, in addition to populations as old as the age of the Universe. Similar solutions are seen for the spectrum coming from the galaxy aperture, which is taken from regions of the slit well outside ( $\left.\geq R_{\mathrm{tid}}\right)$ the central GC aperture. The recovered solutions for the galaxy light also indicate a generally declining SFR superimposed on several local enhancements at intermediate (3-5 Gyr) and young ( $\leq 1 \mathrm{Gyr}$ ) ages. These complex stellar populations are not unexpected given the morphology of the Pegasus CMD in C17, which indicated a spread in stellar population ages.

Given the clear overlap in resolved stellar populations visible in figs 2 and 3 of $\mathrm{C} 17$ it is not surprising that the recovered GC and neighbouring Pegasus spectra show qualitatively similar stellar populations in the full spectral fitting analysis. The resolved star count radial profiles in $\mathrm{C} 17$ show that the old stellar populations (traced by bright giant and horizontal branch stars), are present in all regions of Pegasus, but show a marked increase within the GC's halflight radius. In contrast, the intermediate-age red clump and young main-sequence stars exhibit a slowly increasing density profile with decreasing radius, with no evidence of local enhancement over the Pegasus population within the area of the GC.

This comparison highlights the difficulty of disentangling genuine young populations from integrated light spectra of NSCs, when the host galaxy also has young populations (which naturally will also increase in density in the galaxy's underlying central disc/spheroid). In the central region occupied by the cluster we should have contributions from the old star cluster plus Pegasus, and indeed we see this contamination in the recovered SFH, which is in excellent agreement to what was found in $\mathrm{C} 17$ analysing the spatial distribution of main-sequence, horizontal branch and red clump stars. In the case of Pegasus, the ability of the resolved star catalogue to discriminate the radial behaviour of different stellar populations is a crucial tool to aid the interpretation of the star cluster formation history.

Sufficiently deep resolved CMDs of the NSC and host are not possible in galaxies beyond the Local Group. In those cases, Fig. 3 illustrates the necessity of at minimum having a comparison spectrum from an annulus just outside the NSC, to serve as an estimate of the underlying galaxy stellar populations in which the NSC is embedded. Without this comparison spectrum, any account- 
Pegasus
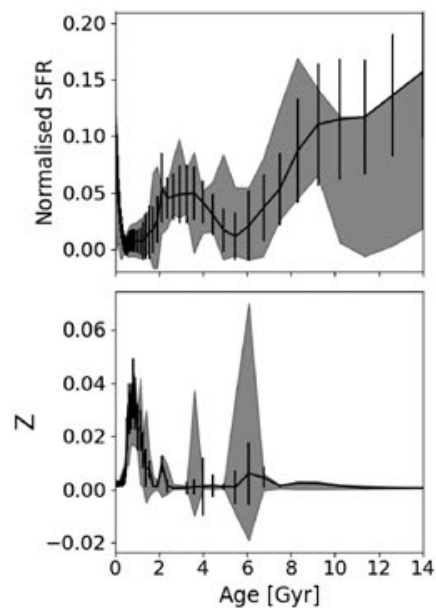

GC



GC

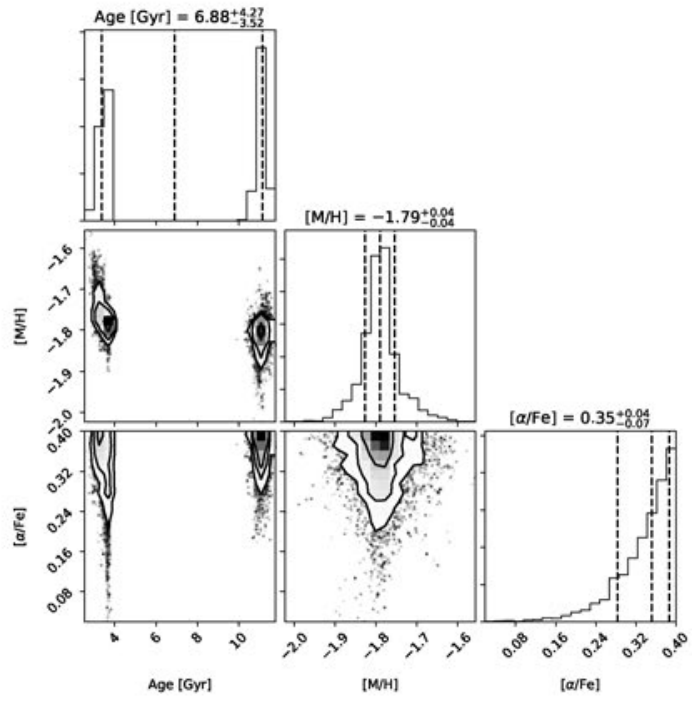

Figure 3. Left: recovered mass fractions from full spectral fitting of Pegasus with STECKMAP. Shaded area shows uncertainty in the solutions for SFR and metallicity at fixed age. Centre: same but for the recovered stellar populations of the GC spectrum. Right: line index-based age and metallicity indicators for the GC derived from the same spectra show good agreement with the full spectral fitting and photometric results. The GC population, after decontamination from the embedded host galaxy light, shows an old metal-poor stellar population.

ing of the NSC's age distribution from full spectral fitting may have an ambiguous physical interpretation due to contamination of disc stellar populations superimposed over top of the NSC. This challenge can remain even when the photometric surface brightness profiles suggests the NSC should dominate the total light in any central spectrum - as young populations from the galaxy would still be easily recovered by modern full spectral fitting codes due to their unique spectral features relative to an old population - even if the former contributes only a small fraction of the total stellar light.

In summary, the separate analysis of the resolved CMD from C17 and integrated spectra of the cluster and Pegasus, allows us to conclude that Pegasus has a mix of stars over all age ranges, while the GC is mainly formed by old metal-poor stellar populations.

\section{ORBITAL INFALL AND EVOLUTION MODELS}

To understand the scenarios in which the GC could have arrived in or passed through its current $3 \mathrm{D}$ galactocentric location, we ran a suite of numerical orbit integration models. For a given host galaxy potential and GC mass, these models calculate the object's orbital evolution including dynamical friction and dissipative mass loss for the GC.

We follow the prescriptions of Petts et al. (2016) for dynamical friction acceleration, which has been well calibrated against $N$-body simulations, and relaxes classical assumptions in the Chandrasehkar formula in order to capture the effect of core stalling by more accurately parametrizing the Coulomb logarithm and velocity distribution terms. The dynamical friction force in this parametrization depends heavily on the density profile of the host galaxy and the distribution function of the stars and DM at various radii.

As we know only coarse information about the host galaxy (its total stellar mass and size), we run $10^{4}$ orbital realizations, each time allowing the parameters of the galaxy potential to vary, so as to test the impact of a different DM and stellar distribution on the results.

For every trial, we randomly draw the total stellar mass of the host galaxy from within its observed (normal) uncertainties. This in turn sets the galaxy's total virial mass via the stellar to halo mass relations of Leauthaud et al. (2012), from which we sample their $1 \sigma$ scatter in $M_{*, \text { gal }} / M_{\text {vir }}$. Given this virial mass, we next sample the redshift zero halo mass-concentration relation and scatter of Dutton \& Macciò (2014) to set the DM halo concentration.

We model the galaxy's DM halo with a 'core NFW' profile, which Read, Agertz \& Collins (2016) show accurately reproduces the potential changes in DM profiles of simulated galaxies as they transition from a cusp to a core, due to baryonic feedback effects. These DM profiles are parametrized in terms of a total mass $M_{\mathrm{vir}}$, core radius $R_{\mathrm{c}}$, and a shape parameter $0 \leq \alpha \leq 1$ - where 0 represents an NFW cusp, and 1 a core.

$\rho_{\mathrm{cNFW}}(r)=f^{\alpha} \rho_{\mathrm{NFW}}+\frac{\alpha f^{\alpha-1}\left(1-f^{2}\right)}{4 \pi r^{2} r_{\mathrm{c}}} M_{\mathrm{NFW}}$
$f=\left\{\tanh \left(\frac{r}{r_{\mathrm{c}}}\right)\right\}^{\alpha}$

We uniformly sample $\alpha$ between 0 and 1 . Following the correlations seen described in Read et al. (2016), we give the galaxy in each trial a core radius of $R_{\mathrm{c}} \sim 1.75 R_{\mathrm{e}}$, using a normal distribution about the measured half-light radius of the galaxy, with $\sigma_{\mathrm{R}_{\mathrm{c}}}=50 \mathrm{pc}$. The galaxy's stellar distribution is added to the total potential as a Sérsic profile using the best-fitting observed surface density profile (with $n_{\mathrm{S}}=1$ ).

The starting distance $D_{\mathrm{GC}}$, i of the $\mathrm{GC}$ is uniformly drawn from 1 to $2000 \mathrm{pc}$ (nearly four times the galaxy's current effective radius), and each starting orbit can have an orbital circularity from $0 \leq \eta \leq 1$ - though we bias the trials to 'in situ formation scenarios' for the GC, by sampling uniformly in eccentricity $\left(0 \leq e=\sqrt{1-\eta^{2}} \leq 1\right)$.

The GC mass in each trial is computed by normally sampling the observed mean absolute magnitude of the GC and its observed magnitude uncertainty $\left(\delta M_{\mathrm{V}}=0.16 \mathrm{mag}\right)$. The absolute magnitude 
is converted to a stellar mass estimate by assuming a normal distribution around $M / L=1.5$ with $\sigma_{\mathrm{M} / \mathrm{L}}=0.33$ in each trial. The combined scatter on these masses is larger than the expected mass loss due to stellar evolution alone, and so we do not incorporate this explicitly into the initial GC mass that we use.

The initial GC structural properties in each simulation are normally sampled from the observed values and uncertainties in the half-light radius, concentration, and distance - together which also yield a realization of the present-day tidal radius, $R_{\mathrm{tid}}$. For every trial, the theoretical Jacobi radius $\left(R_{\mathrm{J}} \sim D_{\mathrm{GC}}\left(M_{\mathrm{GC}} / M_{\mathrm{enc}}\right)^{1 / 3}\right)$ is computed locally at every radius in the galaxy potential - allowing us to understand the structural properties of the GC if it were born in that specific tidal environment.

With the host galaxy potential, GC properties and orbital eccentricity specified, we numerically integrate the orbits accounting for both gravitational and dynamical friction accelerations. The numerical integration is done with an adaptive Adams-BashforthMoulton method, originally coded by Craig Markwardt ${ }^{4}$ to provide a linear multistep explicit method of solving the ordinary differential equations. In each trial we record the characteristic dissipation times (Lamers et al. 2005) and Jacobi radii due in the presence of an external tidal field, at the galactocentric birth distance $\left(T_{\text {diss, }}, R_{\mathrm{J}, \mathrm{i}}\right)$, and at the limit to the $3 \mathrm{D}$ galactocentric present-day location $\left(T_{\mathrm{diss}, 0}\right.$, $R_{\mathrm{J}, 0) \text {. }}$

If an orbital trial is born in, or enters due to dynamical friction, the present-day volume of $D_{3 \mathrm{D}} \leq 86 \mathrm{pc}$ where the GC is observed in Pegasus, we record the time of entry due to dynamical friction $\left(T_{\mathrm{DF}}\right)$, final pericentric radius $\left(D_{\mathrm{per}, \mathrm{f}}\right)$, and the velocity at pericentre $\left(V_{\mathrm{GC}, \text { per }}\right)$. We define the excess time the GC has spent in this central region as $\Delta T=T_{\mathrm{GC}}-T_{\mathrm{DF}}$, where $T_{\mathrm{GC}}$ is the age of the GC in that trial (normally sampled about the observed age, $10 \mathrm{Gyr}$ with $\sigma_{\text {age }}=3 \mathrm{Gyr}$ ). For each trial's intrinsic deprojected velocity in this region, we randomly project it 1000 times at different orbital phases and inclinations and record these velocities relative to the galaxy, $\Delta V=V_{\mathrm{GC}, \text { per }}-V_{\mathrm{gal}}$.

\subsection{Characteristics of successful orbits}

Given the observed present day location of the GC in the centre of the galaxy, we wish to understand: (1) whether it has undergone dynamical friction-driven orbital decay and is at rest with respect to the galaxy ('inspiral'), (2) whether it was born in the centre of the galaxy and survived there until present ('in situ'), or (3) whether it is simply passing through pericentre on an eccentric orbit ('fly-by').

Our orbital simulations allow us to test the feasibility of the three scenarios, taking into account the relevant evolutionary timescales for the GC in all cases. For the inspiral scenario, we define three classes of solutions for GCs that initially were formed outside the centre: trials where the GC arrives to the galaxy centre within its lifetime, trials where it arrives and survives dissipation in the central galactic tidal field, and trials where it arrives, survives, and retains an extended structure consistent with the observed GC concentration and tidal radius. We similarly subdivide the solutions for the fly-by and in situ birth scenarios in the same way.

Fig. 4 shows the orbital evolution of trials that meet these various criteria. The top panels show that there are solutions which can arrive or pass through the galaxy centre at a range of epochs perhaps not surprising given the large variety of orbits and host potentials we sample. However, we must also understand whether

${ }^{4}$ https://www.physics.wisc.edu/ craigm/idl/idl.html

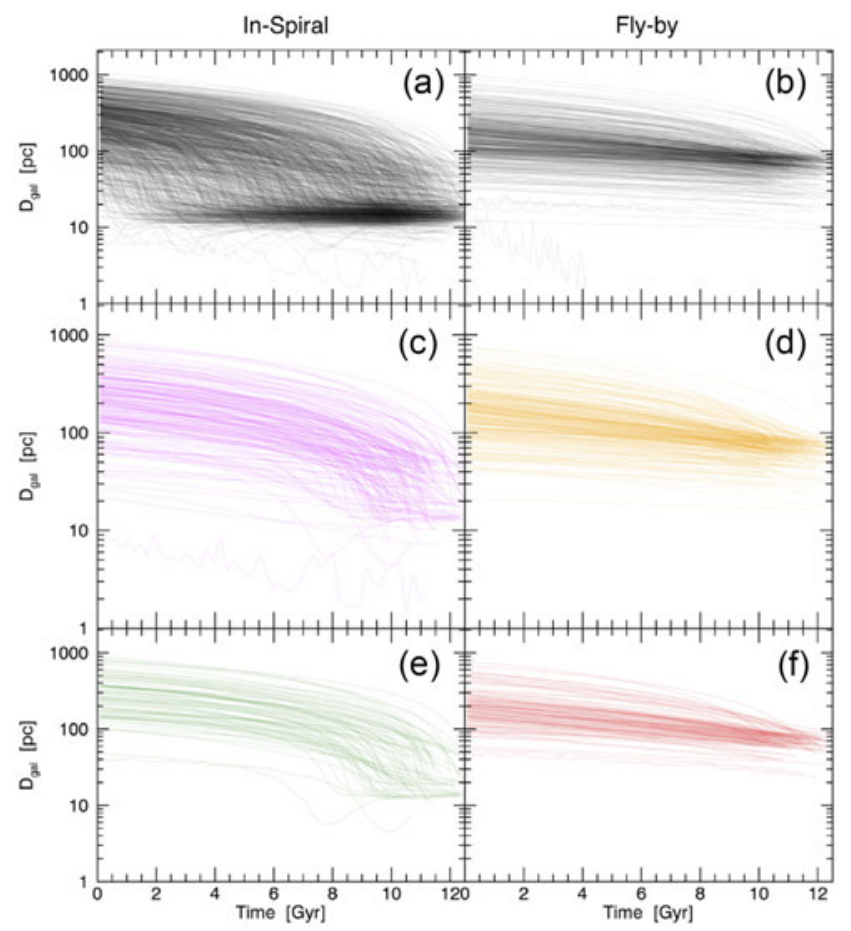

Figure 4. Phase-normalized galactocentric distance versus time, for different subsets of the GC orbital evolution simulations. Left-hand column shows from top to bottom: (a) trials where the GC inspirals to the presentday location within the GC's lifetime (black), (c) trials which inspiral and survive dissipative evaporation in the new tidal field (magenta), and (e) trials which inspiral, survive evaporation, and have an initial Jacobi radius comparable to the observed tidal radius (green). Right-hand column shows: (b) trials with eccentric orbits with pericentre which passes through the central region of the galaxy (black), (d) which also survive that passage (orange), and (f) which also had an initial Jacobi radius comparable to or larger than the observed tidal radius (red).

the GC can survive tidal dissolution in its new location until present day.

We consider survival due to evaporative dissipation for two limiting cases: if the dissipation time in the initial birth $\left(D_{\mathrm{gal}, \mathrm{i}}\right)$ tidal field is greater than the age of the GC $\left(T_{\text {diss, } i}>T_{\mathrm{GC}}\right)$, and if the dissipation time in the final central $\left(D_{\text {gal }}=86 \mathrm{pc}\right)$ tidal field, is greater than the time it has been in the central $86 \mathrm{pc}\left(T_{\text {diss, } 0}>\right.$ $\Delta T)$. Both of these are limiting cases - inclusion of concurrent dynamical mass loss of the GC as it in-spirals would only succeed in producing longer dynamical friction times. Note that we take into account orbital eccentricity in this limiting calculation as well. As shown by the magenta orbital tracks in Fig. 4, this survival requirement tends to favour solutions where the GC arrived in the galaxy centre only in the last $\Delta T=1.4 \pm 1 \mathrm{Gyr}$.

The observed present-day tidal radius of the GC is quite extended $\left(R_{\mathrm{tid}} \geq 75 \mathrm{pc}\right)$ - which along with its long half-light relaxation time ( $T_{\text {rel }}=8 \pm 1$ Gyr) offers additional clues into the initial tidal field (and thus galactic location and potential) in which it was born (or at least occupied $T_{\text {rel }} \sim 8$ Gyr ago). Imposing that the initial Jacobi radius of the GC at its initial location is at least as large as the present-day observed tidal radius $\left(R_{\mathrm{J}, \mathrm{i}} \geq R_{\text {tid }}\right)$ further narrows the possible realizations, as shown by the green tracks in Fig. 4 . While extreme compressive tides may play a role in the GC structure, it is unclear that they significantly work to expand the star cluster (e.g. Bianchini et al. 2015; Webb, Patel \& Vesperini 2017). Compressive 


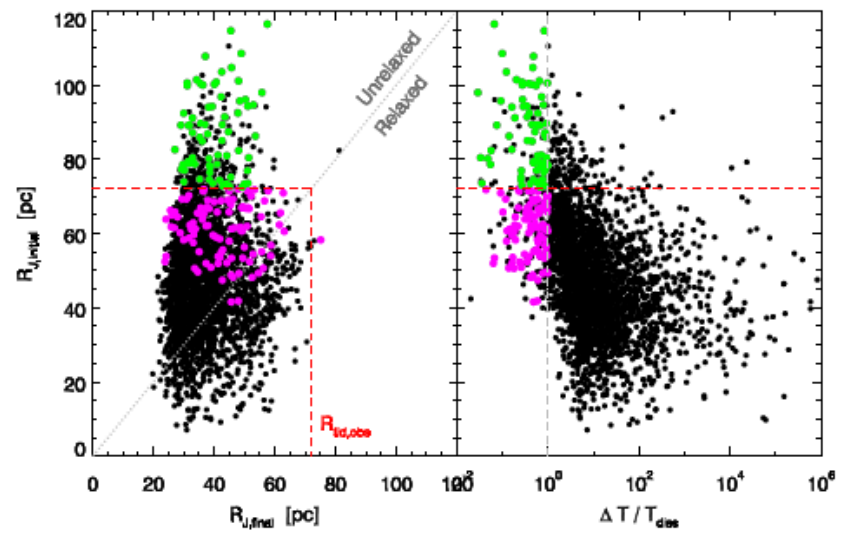

Figure 5. Left: Jacobi radius at the GC's final galactocentric distance versus Jacobi radius at its initial galactocentric distance for trials which inspiral to the central $86 \mathrm{pc}$. The observed lower limit on the tidal radius of the GC is indicated by the red line. Right: summary of the criteria for the successful GC orbital realizations, with initial Jacobi radius plotted against the ratio of the GC's arrival duration in the galaxy centre $(\Delta T)$, relative to its dissipation time there ( $T_{\text {diss }}$ ). Together the survival criteria (magenta points) and structural criteria (green) define a class of favoured solutions.

tides are likely not a relevant evolutionary phase for the Pegasus GC anyway, as Fig. A1 shows that at no point in the orbital evolution, did the GC experience compressive tides in this system, for all of the halo potentials we sampled.

Fig. 5 summarizes the classification of the survival criteria, by plotting the arrival time of the GC in the central region $(\Delta T=$ $T_{\mathrm{GC}}-T_{\mathrm{DF}}$ ) relative to the dissolution time-scale at pericentre, versus the initial Jacobi radius at its birth location in the galaxy $\left(R_{\mathrm{J}, \mathrm{i}}\right)$. While the black trials all manage to arrive at the centre in the GCs lifetime, the magenta points also have arrived there recently enough that they have not dissolved $\left(\Delta T / T_{\text {diss, } 0} \leq 1\right)$. The green trials additionally were initially in a location in the galaxy with a tidal field which produces the observed GC tidal radius $\left(R_{\mathrm{J}, \mathrm{i}} \gtrsim R_{\mathrm{tid}}\right)$.

The last criterion in particular is a crucial constraint on the GC's formation and evolution given that the typical Jacobi radius at its present location is smaller than the observed tidal radius by at least a factor of three $\left(R_{\mathrm{J}, \mathrm{i}} \lesssim 25 \mathrm{pc}\right.$ and $\left.R_{\mathrm{tid}} \geq 75 \mathrm{pc}\right)$. The importance of this constraint can be understood from Fig. 6 where we show the initial Jacobi radius, excess arrival time in the galaxy centre, and final galactocentric distance as a function of initial galactocentric distance of each trial. The top two panels show that trials which have $R_{\mathrm{J}, \mathrm{i}}$ in agreement with the observed GC tidal radius, also arrived in the centre recently (and thus avoid dissolution in the new stronger tidal field). Both facts are a consequence of the large initial distances (which are in weaker tidal fields and have longer dynamical friction inspiral times). The favoured initial formation/inspiral distances in the bottom panel is $700 \mathrm{pc} \lesssim D_{\text {gal }} \lesssim$ $2000 \mathrm{pc}$. We caution that the extremes of these ranges, especially the upper limit, are likely dependent on the number of trials we were to perform, however the median and minimum values of $\sim 1300$ and $\sim 700 \mathrm{pc}$ are likely less sensitive to this aspect of our numerical experiment. In all cases, the arrival time in the centre is less than the present-day GC relaxation time $\left(\Delta T / T_{\text {rel }} \leq 1\right)$, suggesting memory of the initial conditions (structure and tidal field) remains.

From the $10^{4}$ initial trials, the final percentage of solutions which satisfy the various survival and structural criteria are shown in Table 2. These numbers are low in an absolute sense due to the broad, agnostic assumptions about starting distance and host potential that

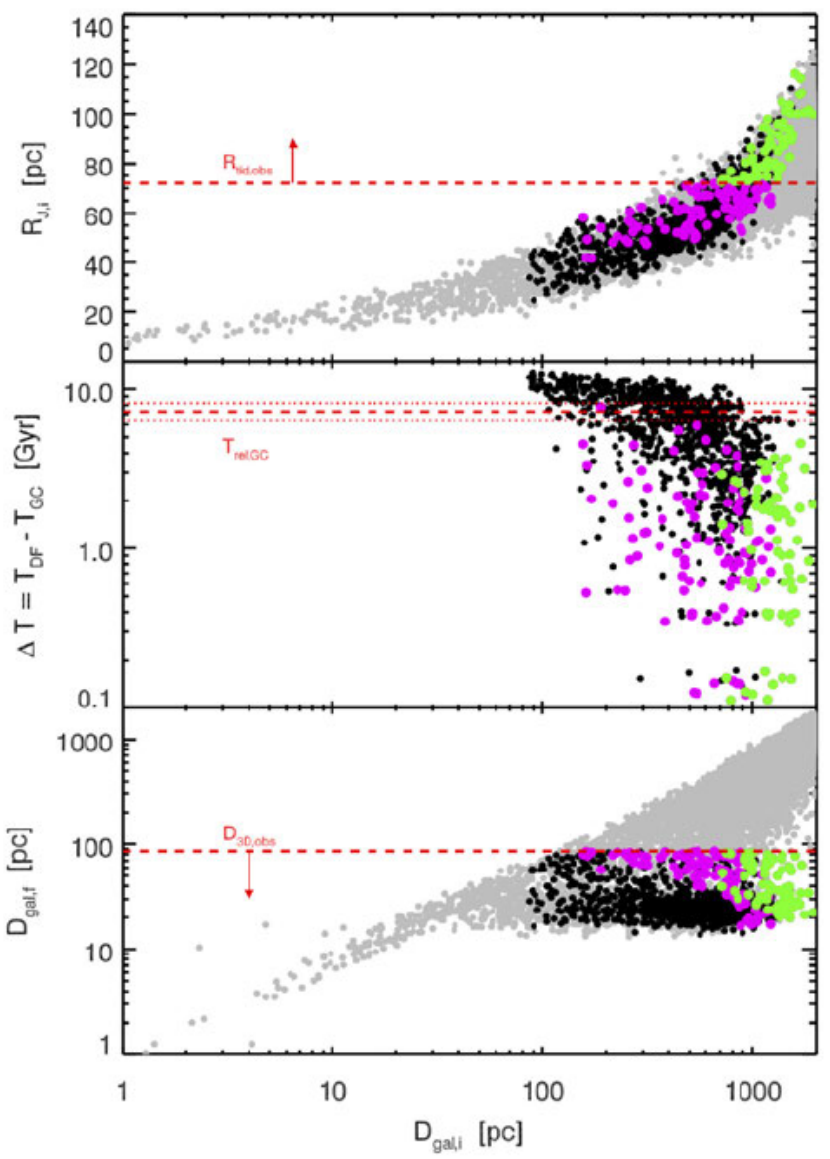

Figure 6. Initial Jacobi radius, excess arrival time in the galaxy centre, and final galactocentric distance as a function of initial galactocentric distance of each trial. Present-day 3D galactocentric distance, relaxation time, and observed tidal radius of the GC are all marked by dashed lines. Trials which inspiral to the galaxy centre within the lifetime of the GC are shown in black, while additional survival and structural constraints are satisfied by the magenta and green points as described in Figs 3 and 4.

Table 2. Statistics of successful GC orbits. Letters correspond to the panels in Fig. 4.

\begin{tabular}{lcc}
\hline Scenario & Criteria & $\begin{array}{c}\text { Percent } \\
\text { of trials }\end{array}$ \\
\hline 'Inspiral' & \\
(a) & Arrive in centre & 11 \\
(c) & Arrive and survive & 1.7 \\
(e) & Arrive, survive w/ $R_{\mathrm{J}} \geq R_{\text {tid }}$ & 0.7 \\
'Fly-by' & Pass through centre \\
(b) & Pass through and survive \\
(d) & Pass through, survive $R_{\mathrm{J}} \geq R_{\text {tid }}$ & 4.6 \\
(f) & Born in centre & 2.4 \\
'In situ' & Born in centre and survive \\
& Born in centre, survive $R_{J} \geq R_{\text {tid }}$ & 4.9 \\
& & 0.3 \\
&
\end{tabular}

we allowed in the simulations. However, trials which successfully reproduce the GC structure and survive dissipation are essentially only able to occur via the fly-by or inspiral mechanisms. Survival alone is about $\sim 10$ times more likely in these scenarios than in the in situ scenarios. Figs 5 and 6 indicate that of the successful 


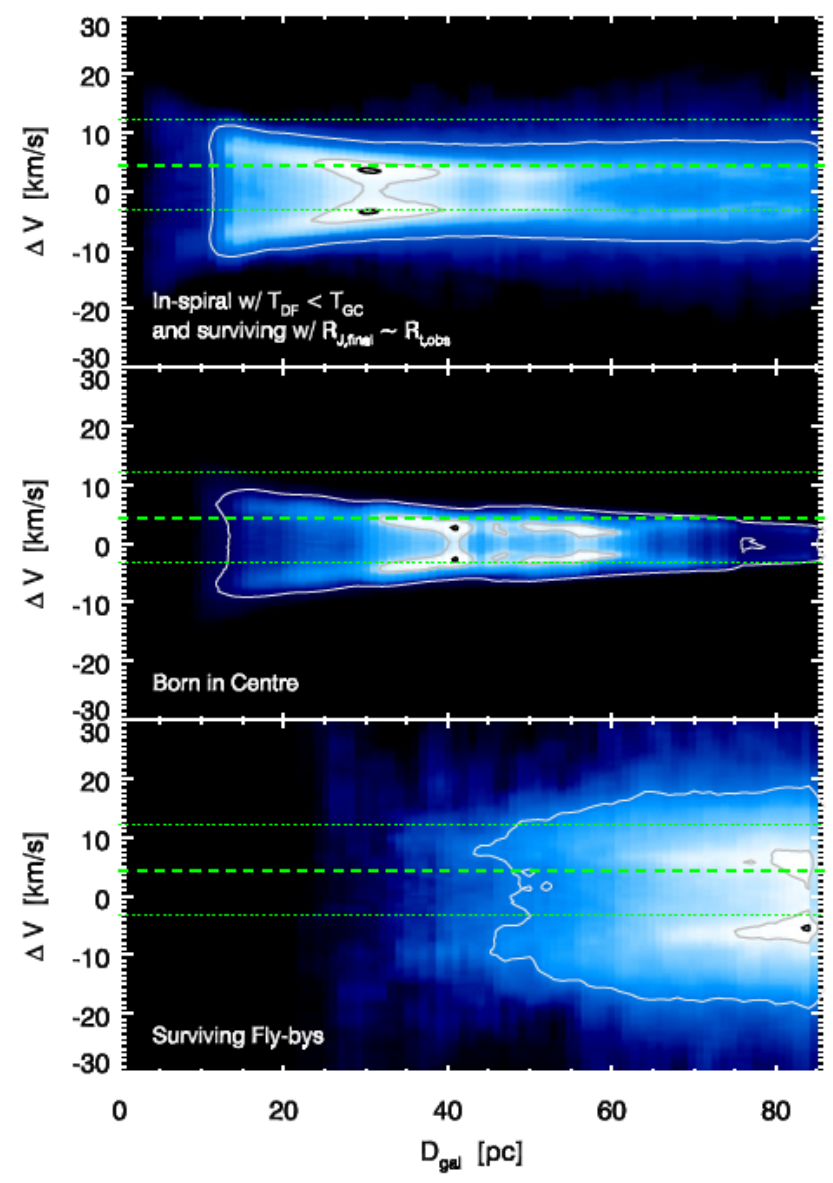

Figure 7. Density distribution of the projected LOS velocities of the GC relative to the host galaxy, for simulated orbits which enter the inner $86 \mathrm{pc}$ of the galaxy. Light grey, grey, and black contours show the $1 \sigma, 2 \sigma$, and $3 \sigma$ levels of the distributions. Top panel shows orbits in which the GC inspiralled due to dynamical friction, survived and has correct structural properties. Middle panel corresponds to GC trials where the GC was born in situ and survived in the central region for the GC's lifetime - however none of these produce a GC with the correct concentration. Bottom panel corresponds to GCs which survive and are passing through the central region of the galaxy on eccentric orbits. In all panels, the green lines show the observed mean (solid) and systematic uncertainty of the GCs velocity relative to Pegasus.

inspiral trials (green), nearly half are expected to have the GC survive dissipation for another 5-10 Gyr.

\subsubsection{Kinematics in the orbital simulations}

We leverage the recorded kinematics in the numerical orbit simulations to understand any constraints that the observed relative velocity may place on the formation history of the GC. The distributions of relative GC LOS velocities in the inner region of the galaxy are shown in Fig. 7 for all classes of successful simulated GC orbital trials. Inspiral solutions in the simulations which arrive, survive, and have the appropriate GC structure, are expected to have on average a lower relative velocity with respect to the host galaxy ( $\Delta V_{\text {ins }} \sim 3 \pm 1 \mathrm{~km} \mathrm{~s}^{-1}$ ), compared to the fly-by scenarios where the GC is passing through on a radial orbit $\left(\Delta V_{\mathrm{fb}} \sim 6 \pm 1 \mathrm{~km} \mathrm{~s}^{-1}\right)$. Scenarios where the GC is born and evolves in the galaxy centre are not kinematically differentiated from the inspiral scenario - though as discussed in Section 5.1, these trials are ruled out based on the much smaller GC tidal radii they predict.
While the best case random error on the observed relative velocities would have been enough to kinematically constrain the two scenarios, as discussed in the preceding subsection, the systematic uncertainty in the recovered relative velocity is roughly twice the precision needed to differentiate the inspiral and flyby scenarios via kinematics alone. Further high $\mathrm{S} / \mathrm{N}$ and spectral resolution observations may allow for an improvement in this however at this point the structural and age limits of the GC provide a stronger constraint on the scenarios than the relative kinematics.

\section{DISCUSSION}

\subsection{What is the origin of the star cluster in Pegasus?}

The observed tidal radius for the star cluster is much larger (a factor of $\gtrsim 3$ ) than the expected Jacobi radius in the central regions of the galaxy. This difference suggests it has not spent its most recent history in the centre of Pegasus, and motivates us to consider what plausible evolutionary scenarios the star cluster could have undergone from its formation until present day.

The preferred orbital trials where the GC arrives in the galaxy centre due to dynamical friction only recently ( $\sim 1 \mathrm{Gyr}$ ago), were preferentially born further out in the galaxy (therefore taking longer to inspiral), and as such have neither dissolved, nor had the memory of that tidal field erased $\left(\Delta T / T_{\text {rel }} \leq 1\right)$. Together these trials offer a plausible explanation for the simultaneous presence of the GC in the central region and its large observed tidal radius $\left(R_{\text {tid }}\right)$. The observed relative velocities of the GC and Pegasus are consistent with the predicted velocities in our orbit integrations for this scenario. Indirect evidence also comes from considering that the current galactocentric distance of the GC is close to where the galaxy enclosed mass equals the GC mass - and thus suggestive of core stalling taking effect in a dynamical friction inspiral scenario (cf. Petts et al. 2016).

While from the observed kinematics we cannot formally rule out scenarios where the GC is passing through the galaxy centre on an eccentric orbit, these solutions typically require an orbital eccentricity of $e_{\text {orb }} \geq 0.8$. Such highly radial orbits may be common for subhaloes (and their GCs) in cosmological $\Lambda$ CDM simulations of hierarchical galaxy assembly, but would be difficult to understand if the GC formed from the rotating gaseous disc of Pegasus at any distance, as the galaxy is extremely isolated and has low enough mass that major baryonic mergers are unlikely to have played a significant role in its evolution. Even after a Hubble time of evolution, stars in similar mass dIrrs show stellar kinematics with $V_{\text {rot }} / \sigma \sim 1$ (e.g. Leaman et al. 2012; Wheeler et al. 2017; Leaman et al. 2017). However, it remains to be seen what the orbit structure of a GC ejected to the low-density region of a dwarf will look like. Subsequent work will explore this with numerical simulations.

The stellar population analysis in C17 and Section 5.3 indicates a predominantly old population for the GC with the young component originating from the galaxy light. If the GC had been embedded in the centre of the dwarf for a lengthy period of time, we might expect a true in situ population to have also formed, as is sometimes observed in NSCs (Kacharov et al. 2018). While there are clear young stellar populations in the field population of the galaxy, the lack of centrally concentrated young stellar populations in the CMD analysis of $\mathrm{C} 17$ is consistent with a recent arrival time (or prevention of star formation in the GC).

In situ formation of the GC in the galaxy centre is also difficult to reconcile with its old age, as multiple dissolution processes would 
unbind the cluster by now - regardless of the galaxy potentials we tried. While in Fig. 6 we computed a limiting survival time for relaxation-driven evaporative dissipation, a more destructive process for star clusters is often tidal threshing in the dense interstellar medium (ISM) in which they formed. Kruijssen (2015) illustrated analytically and with simulations, how the same dense ISM that is required to form massive bound star clusters, is also extremely damaging to them.

The consequence of these findings is that many star clusters may need to quickly migrate out of the dense gaseous environments after their formation. This migration could be driven by resonant dynamical scattering off of non-axisymmetric features, or occur more rapidly via minor mergers. If the GC formed from dense gas in the centre of Pegasus, this scenario suggests it might have needed to migrate to larger distances shortly thereafter in order to survive.

This general scenario outlined by Kruijssen (2015) for the formation of GCs within a region of dense gas and their subsequent migration, concurrently illustrates the difficulty for an in situ birth origin for the GC in the centre of Pegasus and alleviates a potential problem with the orbital infall scenario. Namely were the conditions in the ISM (density and pressure) sufficient to form a GC at the galactocentric distances implied by the tidal radii constraints $(700 \mathrm{pc}$ $\lesssim D_{\text {gal, } \mathrm{i}} \lesssim 2000 \mathrm{pc}$ )? This is important to consider, as these distance ranges include values larger than the current gas and stellar disc of Pegasus.

While the distribution of gas in Pegasus at the epoch of GC formation is unknown, naively we might expect the largest gas densities to be close to the centre of the (more compact) highredshift Pegasus. This is especially the case when one considers the substantial mass fraction of the GC relative to the host galaxy. We discuss the details and limitations of this possible aspect of the GC's early evolution in Appendix B. Despite the uncertainties in how GCs may form, it is plausible that the Pegasus GC could have initially formed in the central region of the galaxy, if indeed this was where the gas densities were the highest. We note that the SFH of Pegasus does not suggest major bursts of star formation after the GC was formed (Cole et al., in preparation) - perhaps indicating that no catastrophic merging events have strongly triggered intense star formation or re-arranged the structure of the galaxy since then.

We stress that the analysis in Appendix B is for $a$ particular model of GC formation. As such it is not meant to be a comprehensive discussion on all possible formation scenarios but rather to motivate further study on this system. Application of the Kruijssen (2015) scenario in Appendix B, then conservatively suggests that the GC could have initially formed in the highly pressurized ISM in the inner regions of the dwarf ( $D_{\text {form }} \lesssim 500$ pc) with a typical GC formation size of $R_{\mathrm{h}} \sim 4 \mathrm{pc}$ and short relaxation time of $\lesssim 1 \mathrm{Gyr}$, and survived by migrating within $\sim 10-100$ Myr to a less dense region of the galaxy.

This short initial relaxation time would crucially allow the cluster to adapt to its new tidal field after it is ejected into the low-density outer regions of Pegasus ( $700 \lesssim D_{\text {gal }} \leq 2000$ pc) sufficiently quickly ( $\sim 10-20$ orbital times, which is $\sim 5-10$ percent of the average inspiral time in our numerical models), such that it can acquire an extended structure (and longer subsequent relaxation time) and still undergo orbital decay slow enough to be in agreement with our inspiral simulations.

While far from a certain or unique description of the evolution of Pegasus and its star cluster, the dynamical time-scales, stellar

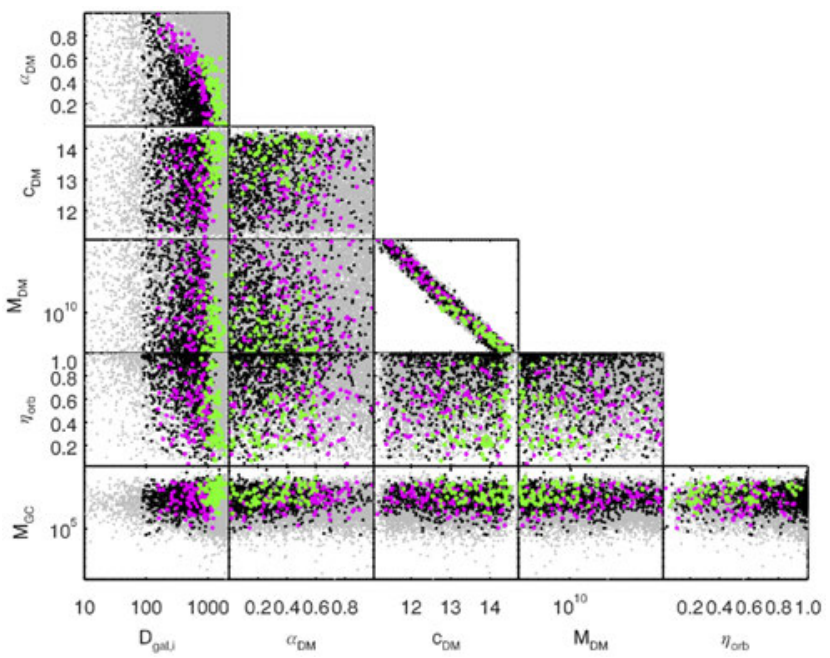

Figure 8. Galaxy halo properties for all orbital evolution trials (grey), with colour coding of successful subclasses of trials the same as in Fig. 5. Shown are the distributions of: inner DM halo slope $\alpha_{\mathrm{DM}}$, halo concentration $c_{\mathrm{DM}}$, total halo mass $M_{\mathrm{DM}}$, orbital circularity $\eta_{\mathrm{orb}}$, GC mass $M_{\mathrm{GC}}$, and initial galactocentric distance $D_{\text {gal, i }}$.

population, and structural properties of this extended GC appear consistent with the following possible sequence of events:

(i) The GC may have been born with a typical size $\left(R_{\mathrm{h}, \mathrm{i}} \sim 4 \mathrm{pc}\right)$ roughly $12 \mathrm{Gyr}$ ago in a dense (possibly central) environment of Pegasus $\left(D_{\text {gal, } \mathrm{i}} \lesssim 500 \mathrm{pc}\right.$ and $\left.\rho_{\text {gas }} \gtrsim 10 \mathrm{M}_{\odot} \mathrm{pc}^{-3}\right)$.

(ii) Within $\lesssim 100 \mathrm{Myr}$, the GC could have been ejected out to a galactocentric distance of at least $\gtrsim 700$ pc during the early assembly of the galaxy.

(iii) The initially short relaxation time $\left(T_{\text {rel, }} \leq 1 \mathrm{Gyr}\right)$ would allow the GC to respond to the new tidal environment, leading to a larger limiting size (comparable to the Jacobi radii at those distances) and longer relaxation time $\left(T_{\text {rel }} \sim 8 \mathrm{Gyr}\right)$.

(iv) As the GC slowly ( $\left.T_{\mathrm{DF}} \sim 10 \mathrm{Gyr}\right)$ migrates due to dynamical friction back to the galaxy centre, its lengthened relaxation time may preserve the newly reconfigured structural imprint of the weaker tidal field - which we observe today as the GC's small concentration and large tidal radius. Further numerical simulations will help understand the feasibility of this possible scenario.

\subsection{Constraints on the host galaxy dark matter profile}

Fig. 8 shows the distribution of dwarf galaxy halo parameters for the various classes of successful inspiral simulation trials. For the GC to merely arrive and survive in the centre after arrival, there are no strong requirements for the halo profile or orbital eccentricity. The time-scale for infall is to first order, set by the starting position in the host, with minimal dependence on the inner density profile slope (see also Meadows et al. 2020). As shown in Section 5.3 however, there is a clear preference for the optimal solutions which also reproduce the GC structure (green points) to have initial starting positions for the GC of $D_{\text {gal, }} \gtrsim 700 \mathrm{pc}$.

These successful inspiral solutions additionally favour high concentration, lower mass DM haloes $\left(M_{\mathrm{DM}} \simeq 6 \pm 2 \times 10^{9} \mathrm{M}_{\odot}\right.$ and $c \simeq 13.7 \pm 0.6$ ). The DM halo shape parameter $\alpha$ interestingly is restricted to be steeper than $\alpha \leq 0.6$ (where $\alpha=1$ is a fully cored profile in equation 4). This is naively counter-intuitive as 


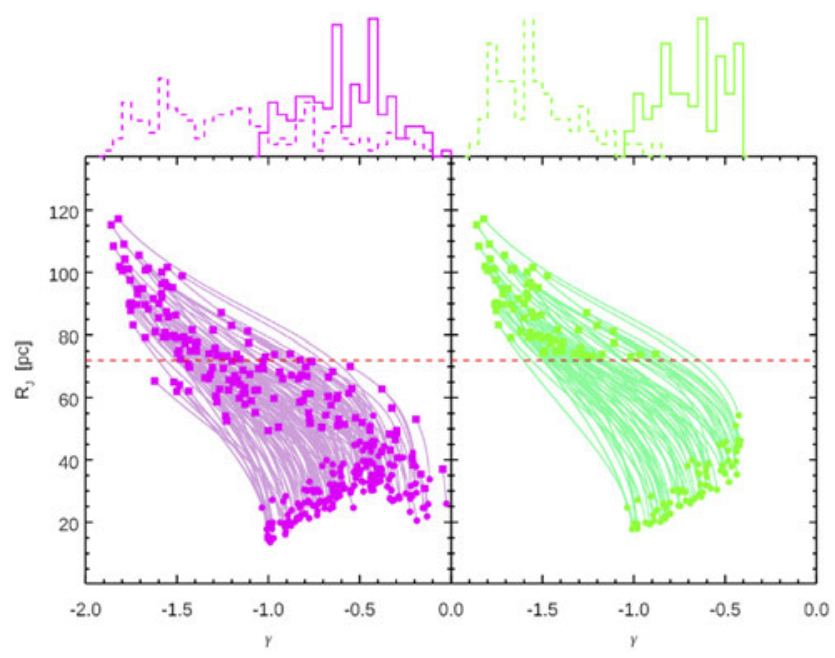

Figure 9. Evolution of the local Jacobi radii for the $\mathrm{GC}$ as a function of the local logarithmic slope of the host galaxy DM density profile $(\gamma)$. Each track is a single GC orbital realization from its birth position (denoted by the squares), to the ending position in the galaxy (circle). Left-hand panel shows that while orbits which inspiral in the necessary time and survive (magenta) have no unique, most-preferred DM halo profile, solutions which also allow for the GC to have an extended structure $\left(R_{\mathrm{J}, \mathrm{i}}>R_{\mathrm{tid}}\right.$; green, right-hand panel) cannot occur in extremely cored haloes. This is evident in the top panels where distributions of the starting (dashed) and final values (solid) for $\gamma$ are shown as histograms.

dynamical friction becomes more efficient for a cored profile, while simultaneously increasing the survival probability by offering a milder tidal field for the GC to reside in.

An explanation comes from considering the tidal field of the GCs at all points along their orbital evolution. In Fig. 9, we show the evolution of the Jacobi radius that the GC would see locally as it evolves from its birth position to the final position, as a function of the local logarithmic slope of the galaxy density profile, $\gamma$. The GC can arrive in the inner region of the galaxy for haloes of essentially any shape, however the left-hand panel of this figure shows that extremely cored profiles have density profiles such that the GC can never form with the necessary tidal radius. The successful trials (green) show values of $-0.9 \leq \gamma \leq-0.5$, with an average value of $\gamma=-0.68 \pm 0.18$.

While the GC survival is more probable for a longer period of time in the cored profiles, these profiles tend to need to be low concentration $(c \leq 12.5)$ in addition to being cored, resulting in the Jacobi radii at the necessary birth distance of the GC being too small. This apparent tension between the cored profiles aiding GC infall and inhibiting formation of extended GCs can be understood from a the following scaling relation argument.

For a galaxy halo with the same total virial mass, the density profile parametrized by $\alpha$ in equation (4) should result in diametric behaviour of the density in a regime inside or outside of the DM halo core radius. As we are considering the star cluster to move beyond the core radius, it is important to normalize to the total virial mass. The top panel of Fig. 10 shows the normalized density profiles as a function of $\alpha$ at fixed radius, or as a function of radius for fixed $\alpha$. Inside the core radius, profiles with high $\alpha$ (cored profiles) show proportionally lower densities as expected. However, for fixed total mass, profiles with $\alpha \gtrsim 0.5$ have densities proportionally higher than cuspy profiles outside the core radius - as the outer regions must contribute progressively more to the total mass.


Figure 10. Top: normalized density profile $\left(\rho(R) / \rho\left(R_{\mathrm{e}}\right)\right.$; left $)$ and enclosed mass $\left(M(R) / M\left(R_{\mathrm{e}}\right)\right.$; right) as a function of DM slope $\alpha$. Bottom: impact of the relative timescale for dynamical friction compared to the simultaneous change in local Jacobi radii as a function of scaled distance in the galaxy (point size) and $\alpha$ (colour bar).

The relative impact of this variable local density on the dynamical friction time and on the initial Jacobi radius of a GC born at some scaled radius $R / R_{\mathrm{e}}$ is:

$\Delta T_{\mathrm{DF}} \equiv T_{\mathrm{DF}} / T_{\mathrm{DF}, \mathrm{e}} \propto \frac{M(R) R^{2}}{M\left(R_{\mathrm{e}}\right) R_{\mathrm{e}}^{2}}$
$\Delta R_{\mathrm{J}} \equiv R_{\mathrm{J}} / R_{\mathrm{J}, \mathrm{e}} \propto\left(\frac{M\left(R_{\mathrm{e}}\right)}{M(R)}\right)^{1 / 3}$

In the bottom panel of Fig. 10, we show the relative change in dynamical friction time versus the corresponding change in initial GC Jacobi radius as a function of the halo inner density profile parameter $\alpha$. This figure quantitatively illustrates the reason why the preferred halo shapes cannot be extremely cored for extended clusters with long relaxation times such as the one in Pegasus. Considering the structural properties together with the orbital decay, may help provide stronger constraints on the host galaxy potential than either property alone (see also Contenta et al. 2018).

We note that the constraints on the slope of the inner DM density profile from our successful orbital trials $(\gamma=-0.68 \pm 0.18)$, are in excellent agreement with independent estimates for Pegasus by Brook \& Di Cintio (2015), who found $\gamma=-0.6$. That work estimated the inner slope using a fitting function for the expected 


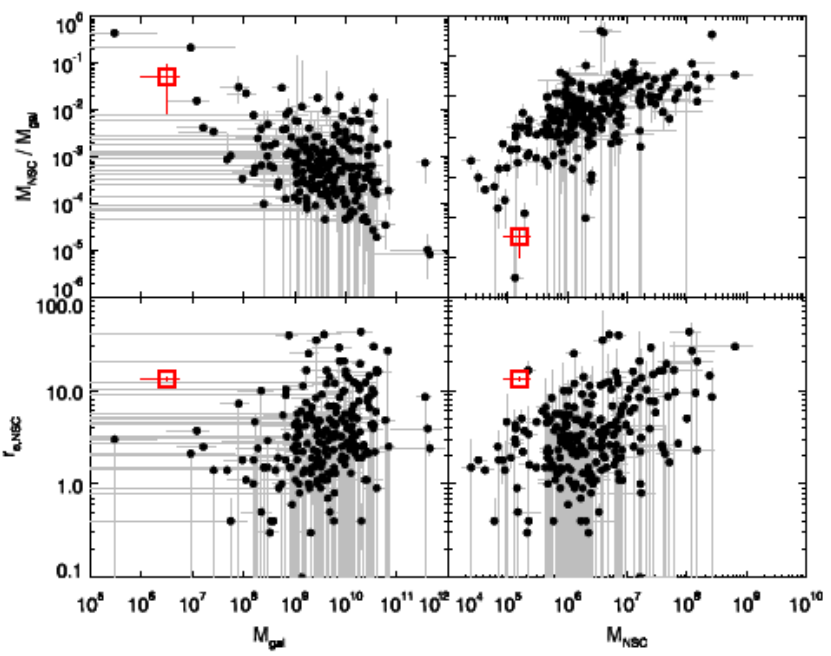

Figure 11. Scaling relations for NSCs from Georgiev et al. (2016). The star cluster in DDO 216 is a notable outlier to NSCs in size-mass diagnostics (red square), and is also the most massive old star cluster relative to its host galaxy mass.

mass-dependent halo shape seen in the cosmological simulations of Di Cintio et al. (2014). The small difference in slopes is likely reconciled due to the smaller halo mass they assumed for Pegasus from abundance matching arguments $\left(M_{\text {vir }}=2.5 \times 10^{9} \mathrm{M}_{\odot}\right.$ compared to our $M_{\text {vir }}=6 \pm 2 \times 10^{9} \mathrm{M}_{\odot}$ ). Galaxies of the mass of Pegasus are not expected to have strong cores according to those simulation studies, which provides strong independent support for our GC-based results.

Studies by Amorisco (2017), Contenta et al. (2018), and Webb \& Vesperini (2018) have shown that the extremely low-mass clusters in the even lower mass dwarfs Eridanus II and And XXV $\left(L_{\text {cluster }} \sim\right.$ $5 \times 10^{3} \mathrm{~L}_{\odot}$ ) require nearly perfect cores in their host galaxies. These studies have elegantly demonstrated the extreme constraints that such fragile clusters provide on the host galaxies, however as noted by Webb \& Vesperini (2018) there will be fundamental differences in the evolution of more massive GCs in massive dwarfs similar to the system studied here. The primary differences with Pegasus appear to be that the more massive and extended GC and stronger host tidal field, require the GC to spend time in diverse regions of the galaxy - leading to the particular constraints on host profile seen here. The second paper in this series (Leaman et al, in preparartion) uses analytic models and $\mathrm{N}$-body simulations to also consider the hydrodynamic formation and destruction processes which are necessary to describe a more complete picture of the evolutionary pathway of low-mass star clusters in faint dwarf galaxies.

\subsection{Understanding the semantic separation between NSCs and} GCs in low-mass dwarfs

While the star cluster in Pegasus appears to be on its way to occupying a permanent home in the centre of the dwarf galaxy, its current half-mass radius $\left(R_{\mathrm{h}} \sim 14 \mathrm{pc}\right)$ places it at odds with NSCs observed in most other types of galaxies. Fig. 11 shows a reproduction of the NSC and host galaxy scaling relations presented in Georgiev et al. (2016), with the Pegasus star cluster appended.

The Pegasus star cluster shows one of the largest fractional masses with respect to its host galaxy (and uniquely so when considering NSCs of similarly old age in that sample - though comparable mass ratios are seen in the NGFS survey by Ordenes-Briceño et al. 2018),



Figure 12. Top: most massive GC as a function of host galaxy stellar mass for Local Group galaxies from literature compilations (see Section 6.3 for references). Upper limits for galaxies where only the total GC system mass was reported are shown as grey arrows. Bottom: GC system total stellar mass as a function of host galaxy stellar mass. Magenta lines show GC system masses forming with a constant fraction of virial mass, following a stellar-to-halo mass relation from Leauthaud et al. (2012). In both panels. the Pegasus star cluster is indicated as the red square and shows values typical for galaxies of its stellar mass. The light blue dotted line indicates the $1: 1$ relation.

with $M_{\mathrm{GC}} / M_{\mathrm{gal}, *} \sim 0.05$. It appears as an even more extreme outlier on the $R_{\mathrm{h}}-M_{\mathrm{NSC}}$ and $R_{\mathrm{h}}-M_{\mathrm{gal}}$ relations - occupying a region with much larger sizes than NSCs with comparable and host masses.

NSCs are thought to have two parallel formation channels: dry merging of inspiralling GCs, together with in situ star formation from accreted gas in the nuclear regions of the galaxy. Given the extended size of the Pegasus star cluster relative to the NSCs in Fig. 11, it appears likely that it would have to undergo net gas inflow to provide the necessary contraction in size, as dry mergers would be unable to increase the density (Perets \& MastrobuonoBattisti 2014; Dutton et al. 2016, though see Capuzzo-Dolcetta \& Miocchi 2008).

In contrast to its anomalous location in the NSC diagrams, the Pegasus star cluster is in excellent agreement with several GC-host galaxy scaling relations. In Fig. 12, we show that the Pegasus GC lays within the range of typical values for the maximum GC mass versus host galaxy stellar mass relation defined by Local Group galaxies in Forbes et al. (2018). The bottom panel of Fig. 12 shows that the Pegasus GC also follows the total mass in GCs versus 
host galaxy stellar mass seen in the same Local Group galaxies, as well as field and cluster galaxies from Peng et al. (2006), Spitler \& Forbes (2009), Harris, Harris \& Alessi (2013), Baldassare et al. (2014), Amorisco et al. (2018), Beasley et al. (2019), and Prole et al. (2019). We note that some of these studies only report the total number of GCs, and in this case we have assumed a typical GC mass of $2 \times 10^{5} \mathrm{M}_{\odot}$. If not reported in the original studies we show a 0.5 dex error bar on the GC system mass.

The shape of this scaling relation is typically interpreted as the GC system mass forming with a fixed fraction of the host galaxy virial mass, and so we also show a prediction of the form $M_{\mathrm{GC} \text {, tot }}=\eta M_{\mathrm{vir}}$, using the stellar-to-halo mass relation of Leauthaud et al. (2012) to convert the virial mass to stellar mass, and $\eta=2.5 \times 10^{-5}$. We refer the interested reader to Forbes et al. (2018) for detailed discussions on sample selection effects and astrophysical effects altering the GC system masses - however here simply note that there are several galaxies with GC system masses comparable to Pegasus at its same host mass.

As discussed recently in El-Badry et al. (2019), the roughly constant fraction of $M_{\mathrm{GC}} / M_{\mathrm{vir}}$ over many orders of galaxy mass is potentially a consequence of the central limit theorem in a mergerdriven $\Lambda \mathrm{CDM}$ galaxy assembly framework. Low-mass systems such as Pegasus are crucially able to provide leverage into the primordial physics of GC formation and evolution, as their total mass in GCs (and to a lesser extent, virial mass) should not have been altered by merger events. That Pegasus and its GC follow the same relation that is driven by stochastic merging in high-mass galaxies (see also Forbes et al. 2018), may lead to better insight to the relation between these two quantities in the context of star cluster formation physics (e.g. Kruijssen 2012).

Continued observational studies of dwarf galaxies in the lowmass regime $\left(M_{\text {vir }} \lesssim 10^{10.5} \mathrm{M}_{\odot}\right)$ where stellar mergers have not strongly contributed to the asymptotic correlation between $M_{\mathrm{GC}^{-}}$ $M_{\text {vir }}$ would be beneficial in large number, in order to ascertain the primordial $M_{\mathrm{GC}}-M_{\mathrm{vir}}$ relation and assess the impact of environmental processing to the host galaxy and its star cluster populations.

\section{SUMMARY}

Using new spectroscopic observations and numerical orbital models, we have studied possible formation and evolutionary pathways for the recently discovered massive $\left(M_{\mathrm{GC}} \geq 10^{5}\right)$, extended $\left(R_{\mathrm{h}} \sim\right.$ $14 \mathrm{pc})$ star cluster in the dwarf galaxy Pegasus. This star cluster presents an unusually large mass relative to its host, and a tidal radius larger than expected for its location in the dwarf galaxy both of which suggests a varied set of tidal environments were involved in, and necessary for, its evolution and survival. Cruciall, we have leveraged strong constraints on the $3 \mathrm{D}$ distance of the GC from differential RR Lyrae variable star analysis of the GC and its host galaxy to help understand the history of this star clusters. The main conclusions of our study are as follows:

(i) Line index and full spectral fitting of the GC and host galaxy confirm it to have a uniquely old and metal-poor stellar population. Crucially, a comparison spectrum of the galaxy and the resolved stellar populations of the HST CMD allow us to disentangle the intrinsic GC populations from the complex SFH of the dwarf galaxy it is embedded in.

(ii) Our kinematic observations indicate the star cluster has a velocity of $\Delta V=3 \pm 4$ (ran.) \pm 8 (sys.) $\mathrm{km} \mathrm{s}^{-1}$ with respect to the galaxy's systemic velocity. (iii) Orbital simulations in host galaxies with a variety of gravitational potentials (spanning from cusped to fully cored) suggest that the GC could have in-spiraled from the galaxy outskirts (700 pc $\leq$ $D_{\text {gal }} \leq 2000 \mathrm{pc}$ and arrived in the centre within the last $1.4 \pm 1 \mathrm{Gyr}$. However systematics in multiple full spectral fitting codes prevent observational kinematic differentiation of scenarios where the GC is simply passing through pericentre on an eccentric orbit, or whether it has arrived at rest in the centre of the galaxy.

(iv) Based simply on the fact of the arrival and survival of the GC at the centre of the galaxy, no detailed DM halo properties can be inferred.However to reproduce the GC's extended structure and low concentration, a galaxy with an inner slope to its DM density profile of $0.9 \leq \gamma \leq-0.5$ and virial mass of $M_{\mathrm{vir}}=6 \pm 2 \times 10^{9} \mathrm{M}_{\odot}$ is preferred. This is in excellent agreement with the inefficient DM core creation seen independently in smoothed particle hydrodynamics simulations for galaxies of this mass (Brook \& Di Cintio 2015).

(v) Consideration of the ISM density required for pressure equilibrium-driven formation of GCs suggests in that scenario the GC may have likely needed to form in the inner $\sim 500$ pc of Pegasus, and then quickly migrate out of the centre of the dwarf to the above mentioned galactocentric distances on time-scales of tens of Myrs.

(vi) The extended size of the GC was therefore likely not intrinsic from birth, but rapidly acquired following this requisite ejection to the dwarf galaxy outskirts, due to its short initial relaxation time ( $T_{\text {rel }} \leq 1 \mathrm{Gyr}$ ).

(vii) The extended GC size set by this new tidal environment was preserved during its subsequent inspiral to the centre of Pegasus, due to the much longer relaxation time associated to the large $R_{\mathrm{h}}$, and is responsible for its anomalous present-day observed size ( $\sim 3$ times larger than the estimated Jacobi radius at its galactocentric location).

(viii) The Pegasus star cluster falls naturally on host galaxy-GC scaling relations, but will require significant structural rearrangement (likely in situ gas accretion to increase its density) in order to make it compatible with properties of NSCs observed in the Local Universe. The object is thus perhaps best defined as a GC currently, until such a transition occurs.

We are potentially witnessing an early stage of NSC formation in Pegasus, with the results hinting towards a possible future scenario in which if it survives, this extended GC could become the nuclei of Pegasus, provided that the tidal environment (and any possible gas inflow and in situ star formation) produce the requisite structural changes. Given the difficulties in determining detailed halo shapes from $\mathrm{HI}$ rotation curves or stellar kinematics (or both; Leung et al., 2019) continued use of GCs as dynamical probes of the host galaxy potential should be prioritized in systems with handfuls of GCs such as WLM, Fornax, NGC 6822, Sextans A/B, and other dwarfs in the Local Group. These systems offer unparalleled access to not only the host galaxy structure, but the efficiency of formation and destruction processes of GCs in systems which have not been significantly rearranged by hierarchical merging in $\Lambda \mathrm{CDM}$ cosmologies.

\section{ACKNOWLEDGEMENTS}

We thank the anonymous referee for a constructive referee report which greatly improved this manuscript. The authors thank Glenn van de Ven, Morgan Fouesneau, Anna Lisa Varri, Gigi Leung, Diederik Kruijssen, Carme Gallart, and Matteo Monelli for useful discussions which improved the manuscript. Dedicated to BL. RL thanks AH. This work was made possible with funding from the Natural Sciences and Engineering Research Council of Canada PDF award and supported by Sonderforschungsbereich SFB 881 
'The Milky Way System' (subproject A7 and A8) of the German Research Foundation (DFG), and Deutscher Akademischer Austauschdienst (DAAD) PPP project number 57316058 'Finding and exploiting accreted star clusters in the Milky Way'. TRL acknowledges support via grants AYA2014-56795-P and AYA2016-77237C3-1-P from the Spanish Government. PB acknowledges financial support from a Canadian Institute for Theoretical Astrophysics (CITA) National Fellowship. MAB and JFB acknowledge support from grant AYA2016-77237-C3-1-P from the Spanish Ministry of Economy and Competitiveness (MINECO) and from the Severo Ochoa Excellence programme SEV-2015-0548.

\section{REFERENCES}

Alfaro-Cuello M. et al., 2019, ApJ, 886, 57

Amorisco N. C., 2017, ApJ, 844, 64

Amorisco N. C., Monachesi A., Agnello A., White S. D. M., 2018, MNRAS, 475,4235

Antonini F., Capuzzo-Dolcetta R., Mastrobuono-Battisti A., Merritt D., 2012, ApJ, 750, 111

Antonini F., Barausse E., Silk J., 2015, ApJ, 812, 72

Aparicio A., Gallart C., Bertelli G., 1997, AJ, 114, 669

Arca-Sedda M., Capuzzo-Dolcetta R., 2017, MNRAS, 464, 3060

Baldassare V. F., Gallo E., Miller B. P., Plotkin R. M., Treu T., Valluri M., Woo J.-H., 2014, ApJ, 791, 133

Bartko H. et al., 2009, ApJ, 697, 1741

Beasley M. A., Trujillo I., 2016, ApJ, 830, 23

Beasley M. A., Romanowsky A. J., Pota V., Navarro I. M., Martinez Delgado D., Neyer F., Deich A. L., 2016, ApJ, 819, L20

Beasley M. A., Trujillo I., Leaman R., Montes M., 2018, Nature, 555, 483

Beasley M. A., Leaman R., Gallart C., Larsen S. S., Battaglia G., Monelli M., Pedreros M. H., 2019, MNRAS, 487, 1986

Bermejo-Climent J. R., et al., 2018, MNRAS, 479, 1514

Bianchini P., Renaud F., Gieles M., Varri A. L., 2015, MNRAS, 447, L40

Brook C. B., Di Cintio A., 2015, MNRAS, 450, 3920

Burstein D., Faber S. M., Gaskell C. M., Krumm N., 1984, ApJ, 287, 586

Cappellari M., 2017, MNRAS, 466, 798

Cappellari M., Emsellem E., 2004, PASP, 116, 138

Capuzzo-Dolcetta R., Miocchi P., 2008, ApJ, 681, 1136

Cervantes J. L., Vazdekis A., 2009, MNRAS, 392, 691

Choksi N., Gnedin O. Y., Li H., 2018, MNRAS, 480, 2343

Cole A. A. et al., 2017, ApJ, 837, 54( C17)

Collins M. L. M. et al., 2009, MNRAS, 396, 1619

Contenta F. et al., 2018, MNRAS, 476, 3124

Di Cintio A., Brook C. B., Dutton A. A., Macciò A. V., Stinson G. S., Knebe A., 2014, MNRAS, 441, 2986

Dutton A. A., Macciò A. V., 2014, MNRAS, 441, 3359

Dutton A. A. et al., 2016, MNRAS, 461, 2658

El-Badry K., Quataert E., Weisz D. R., Choksi N., Boylan-Kolchin M., 2019, MNRAS, 482, 4528

Elmegreen B. G., Efremov Y. N., 1997, ApJ, 480, 235

Falcón-Barroso J. et al., 2006, MNRAS, 369, 529

Falcón-Barroso J., Sánchez-Blázquez P., Vazdekis A., Ricciardelli E., Cardiel N., Cenarro A. J., Gorgas J., Peletier R. F., 2011, A\&A, 532, A95

Feldmeier-Krause A. et al., 2015, A\&A, 584, A2

Forbes D. A., Read J. I., Gieles M., Collins M. L. M., 2018, MNRAS, 481, 5592

Gallagher J. S., Tolstoy E., Dohm-Palmer R. C., Skillman E. D., Cole A. A., Hoessel J. G., Saha A., Mateo M., 1998, AJ, 115, 1869

Genzel R., Eisenhauer F., Gillessen S., 2010, Rev. Mod. Phys., 82, 3121

Georgiev I. Y., Böker T., Leigh N., Lützgendorf N., Neumayer N., 2016, MNRAS, 457, 2122

Gnedin O. Y., Ostriker J. P., Tremaine S., 2014, ApJ, 785, 71

Governato F. et al., 2010, Nature, 463, 203

Guillard N., Emsellem E., Renaud F., 2016, MNRAS, 461, 3620
Harris W. E., 2009, ApJ, 699, 254

Harris W. E., Harris G. L. H., Alessi M., 2013, ApJ, 772, 82

Herwig F., VandenBerg D. A., Navarro J. F., Ferguson J., Paxton B., 2012, ApJ, 757, 132

Hoessel J. G., Mould J. R., 1982, ApJ, 254, 38

Huxor A. P., Tanvir N. R., Irwin M. J., Ibata R., Collett J. L., Ferguson A. M. N., Bridges T., Lewis G. F., 2005, MNRAS, 360, 1007

Hwang N., Park H. S., Lee M. G., Lim S., Hodge P. W., Kim S. C., Miller B., Weisz D., 2014, ApJ, 783, 49

Kacharov N., Neumayer N., Seth A. C., Cappellari M., McDermid R., Walcher C. J., Böker T., 2018, MNRAS, 480, 1973

Kelson D. D., 2003, PASP, 115, 688

Kirby E. N., Bullock J. S., Boylan-Kolchin M., Kaplinghat M., Cohen J. G., 2014, MNRAS, 439, 1015

Koleva M., Prugniel P., Bouchard A., Wu Y., 2009, A\&A, 501, 1269

Kruijssen J. M. D., 2012, MNRAS, 426, 3008

Kruijssen J. M. D., 2015, MNRAS, 454, 1658

Kruijssen J. M. D., Pfeffer J. L., Reina-Campos M., Crain R. A., Bastian N., 2019, MNRAS, 486, 3180

Laevens B. P. M. et al., 2014, ApJ, 786, L3

Lamers H. J. G. L. M., Gieles M., Bastian N., Baumgardt H., Kharchenko N. V., Portegies Zwart S., 2005, A\&A, 441, 117

Larsen S. S., Brodie J. P., 2000, AJ, 120, 2938

Larsen S. S., Strader J., Brodie J. P., 2012, A\&A, 544, L14

Leaman R., 2012, AJ, 144, 183

Leaman R. et al., 2012, ApJ, 750, 33

Leaman R., VandenBerg D. A., Mendel J. T., 2013, MNRAS, 436, 122

Leaman R. et al., 2017, MNRAS, 472, 1879

Leauthaud A. et al., 2012, ApJ, 744, 159

Leung G. Y. C., Leaman R., van de Ven G., Battaglia G., 2019, preprint (arXiv:1911.09167)

Leung Y. C., Leaman R., Battaglia G., van de Ven G., Brooks A., Penarrubia J., Venn K., 2019, MNRAS, submitted

Levin Y., Beloborodov A. M., 2003, ApJ, 590, L33

Loose H. H., Kruegel E., Tutukov A., 1982, A\&A, 105, 342

Lora V., Grebel E. K., Sánchez-Salcedo F. J., Just A., 2013, ApJ, 777, 65

Lu J. R., Ghez A. M., Hornstein S. D., Morris M. R., Becklin E. E., Matthews K., 2009, ApJ, 690, 1463

Mackey A. D. et al., 2006, ApJ, 653, L105

Martín-Navarro I., Vazdekis A., Falcón-Barroso J., La Barbera F., Yıldırım A., van de Ven G., 2018, MNRAS, 475, 3700

Maschberger T., Kroupa P., 2011, MNRAS, 411, 1495

Massari D., Koppelman H. H., Helmi A., 2019, A\&A, 630, L4

McConnachie A. W., 2012, AJ, 144, 4

McConnachie A. W., Venn K. A., Irwin M. J., Young L. M., Geehan J. J., 2007, ApJ, 671, L33

Meadows N., Navarro J. F., Santos-Santos I., Benitez-Llambay A., Frenk C., 2020, MNRAS, 491, 3336

Milosavljević M., 2004, ApJ, 605, L13

Misgeld I., Hilker M., 2011, MNRAS, 414, 3699

Navarro J. F., Frenk C. S., White S. D. M., 1997, ApJ, 490, 493

Ocvirk P., Pichon C., Lançon A., Thiébaut E., 2006a, MNRAS, 365, 74

Ocvirk P., Pichon C., Lançon A., Thiébaut E., 2006b, MNRAS, 365, 46

Ordenes-Briceño Y. et al., 2018, ApJ, 860, 4

Orkney M. D. A., Read J. I., Petts J. A., Gieles M., 2019, MNRAS, 488, 2977

Peng E. W. et al., 2006, ApJ, 639, 95

Perets H. B., Mastrobuono-Battisti A., 2014, ApJ, 784, L44

Pérez I. et al., 2017, MNRAS, 470, L122

Petts J. A., Read J. I., Gualandris A., 2016, MNRAS, 463, 858

Prole D. J. et al., 2019, MNRAS, 484, 4865

Read J. I., Agertz O., Collins M. L. M., 2016, MNRAS, 459, 2573

Renaud F., 2010, PhD thesis, Universite de Strasbourg

Ruiz-Lara T. et al., 2015, A\&A, 583, A60

Ruiz-Lara T. et al., 2016, MNRAS, 456, L35

Ruiz-Lara T. et al., 2017, A\&A, 604, A4

Ruiz-Lara T. et al., 2018a, MNRAS, 478, 2034

Ruiz-Lara T. et al., 2018b, A\&A, 617, A18 
Sánchez-Blázquez P. et al., 2006, MNRAS, 371, 703

Sánchez-Blázquez P., Ocvirk P., Gibson B. K., Pérez I., Peletier R. F., 2011, MNRAS, 415, 709

Sánchez-Blázquez P. et al., 2014, A\&A, 570, A6

Sarzi M. et al., 2006, MNRAS, 366, 1151

Schinnerer E., Böker T., Emsellem E., Lisenfeld U., 2006, ApJ, 649, 181

Schinnerer E., Böker T., Meier D. S., Calzetti D., 2008, ApJ, 684, L21

Spitler L. R., Forbes D. A., 2009, MNRAS, 392, L1

Tonini C., 2013, ApJ, 762, 39

Tsatsi A., Mastrobuono-Battisti A., van de Ven G., Perets H. B., Bianchini P., Neumayer N., 2017, MNRAS, 464, 3720

van Dokkum P. G., 2001, PASP, 113, 1420

Vazdekis A., Sánchez-Blázquez P., Falcón-Barroso J., Cenarro A. J., Beasley M. A., Cardiel N., Gorgas J., Peletier R. F., 2010, MNRAS, 404, 1639

Vazdekis A. et al., 2015, MNRAS, 449, 1177

Webb J. J., Vesperini E., 2018, MNRAS, 479, 3708

Webb J. J., Sills A., Harris W. E., Gómez M., Paolillo M., Woodley K. A., Puzia T. H., 2016, MNRAS, 460, 2129

Webb J. J., Patel S. S., Vesperini E., 2017, MNRAS, 468, L92

Weisz D. R. et al., 2016, ApJ, 822, 32

Wheeler C., Pace A. B., Bullock J. S., Boylan-Kolchin M., Onorbe J., Fitts A., Hopkins P. F., Keres D., 2017, MNRAS, 465, 2420

Worthey G., Faber S. M., Gonzalez J. J., Burstein D., 1994, ApJS, 94, 687

Young L. M., van Zee L., Lo K. Y., Dohm-Palmer R. C., Beierle M. E., 2003, ApJ, 592, 111

Zhu L. et al., 2016, MNRAS, 462, 4001

\section{APPENDIX A: COMPRESSIVE TIDAL FIELDS IN THE REALIZATIONS}

In Fig. A1, we illustrate the tidal history of the cluster in the various inspiral realizations. These quantify the level of expansive or compressive tides in the local tidal field (cf. Renaud 2010).

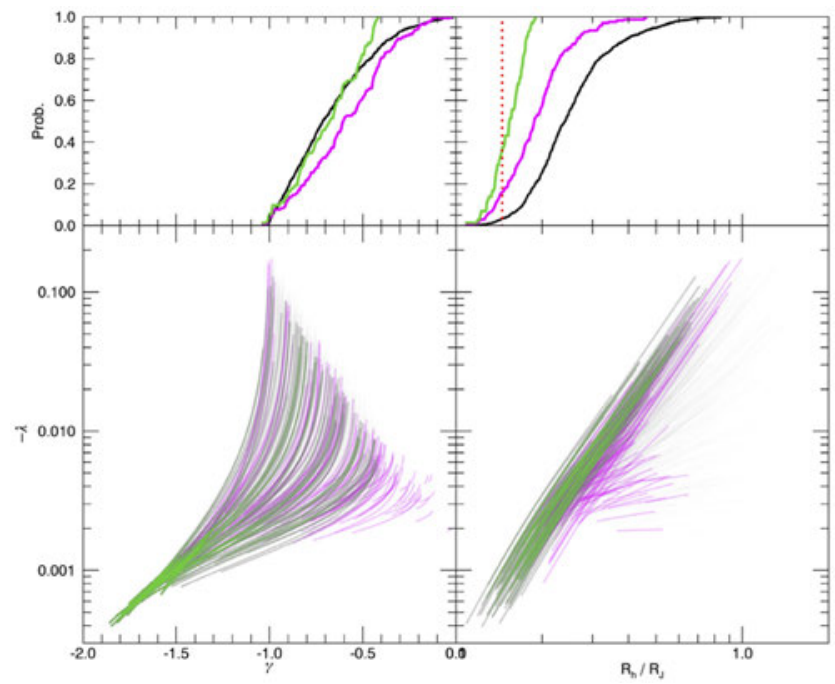

Figure A1. Evolution of the local tidal field strength $(-\lambda)$ as a function of the logarithmic slope of the host galaxy density profile $(\gamma)$, and tidal filling factor $\left(R_{\mathrm{h}} / R_{\mathrm{J}}\right)$. Each track shows a single GC orbital realization for the successful realizations which infall (black), infall and survive (magenta), and infall, survive, and have the observed GC structure (green). The GC likely stayed close to tidally filling $\left(R_{\mathrm{h}} / R_{\mathrm{J}} \sim 0.145\right)$ in the galaxy outskirts, as the cumulative distribution of the filling factor at birth shows in the top right panel.

\section{APPENDIX B: ESTIMATING PROPERTIES OF POSSIBLE FORMATION ENVIRONMENTS FOR THE GC}

Much of the detailed small-scale physics of GC formation is uncertain, however a coarse estimate of the gas density required to form a $\mathrm{GC}$ of a given mass $\left(M_{\mathrm{GC}}\right)$ and size $\left(R_{\mathrm{h} \text {, form }}\right)$ follows from arguments presented in Elmegreen \& Efremov (1997), whereby the kinematic density of the GC might form in some relative pressure equilibrium with the galactic disc mid-plane pressure:

$M_{\mathrm{GC}} R_{\mathrm{h}, \text { form }}^{2} \simeq\left(\frac{\pi}{2} \Sigma_{\mathrm{gas}}\left(\Sigma_{\mathrm{gas}}+\frac{\sigma_{\mathrm{gas}}}{\sigma_{*}} \Sigma_{*}\right)\right)^{1 / 2}$.

In the context of this specific scenario, we can ask what types of gas densities could be needed to form a star cluster with properties similar to the one in Pegasus, and what typical locations in an idealized galaxy these densities may have occurred at $\left(D_{\mathrm{form}} \propto\left(M_{\mathrm{gas}} / \rho_{\mathrm{gas}}\right)^{1 / 3}\right)$. For a fixed $M_{\mathrm{GC}}$, equation (B1) predicts what size star cluster could form in a galaxy disc with gas density of $\rho_{\text {ISM }}$ at some distance.

It is impossible to precisely know the density distribution of molecular gas in the proto-galaxy at redshifts 2-6 when the star cluster formed. However, there are physically motivated limiting cases which must hold, which we can use to constrain the free parameters in this redshift independent framework:

(i) $M_{\mathrm{gas}}$ : for the conservative scenario of 100 per cent star formation efficiency (SFE) and no gas lost to outflows, at minimum $M_{\text {gas }} \simeq$ $M_{*}=6 \times 10^{6} \mathrm{M}_{\odot}$ must have been available in Pegasus. Increasing this by an order of magnitude would only increase the predicted minimum formation distance by a factor of $\sim 2$. We conservatively adopt a gas mass of $M_{\text {gas }}=10^{8} \mathrm{M}_{\odot}$, which is almost two orders of magnitude more than the present-day gas mass, and given the SFH of Pegasus would correspond to an SFE of $\sim 1$ per cent. Significantly larger values for the total gas mass begin to exceed the likely virial mass for Pegasus.

(ii) $\rho_{\text {gas }}(D)$ : we assume a limiting case where the total gas mass was distributed with uniform density out to some radius $D_{\text {form }}$. If the gas was distributed in an exponential disc, as is commonly observed, the predicted formation distance would be even closer to the galaxy centre than this conservative limit.

(iii) $\Sigma_{\text {gas }}(D)$ : to convert an arbitrary $\rho_{\text {gas }}$ to a surface mass density, we assume an upper limit to the molecular gas scale height of $h=300 \mathrm{pc}$. This is far larger than observed molecular disc scale heights in galaxies of this mass and also therefore the giant molecular cloud (GMC) sizes.

(iv) $\sigma_{\text {gas }} / \sigma_{*}$ : the ratio of gas to stellar velocity dispersion is expected to evolve over time and vary from galaxy to galaxy, however a suitable upper limit of $\sigma_{\text {gas }} / \sigma_{*} \simeq 1$ seen observationally in Leaman et al. (2017, and expected from theoretical arguments) can be adopted.

For a known GC mass and the above assumptions in this scenario, equation (B1) can be used to formulate a coarse estimate of the formation size of the $\mathrm{GC}, R_{\mathrm{h}}$, form as a function of the galactocentric distance it formed at, $D_{\text {form }}$.

The bottom panel of Fig. B1 shows this conservative estimate on the upper limit to the GC's formation distance, as a function of the initial GC size, for different arbitrary volumetric gas densities $\left(\rho_{\text {gas }}\right)$. Even for these conservative cases, a GC with density like the one in Pegasus would require a formation distance of $D_{\text {form }} \lesssim$ $200 \mathrm{pc}$ in the galaxy. 


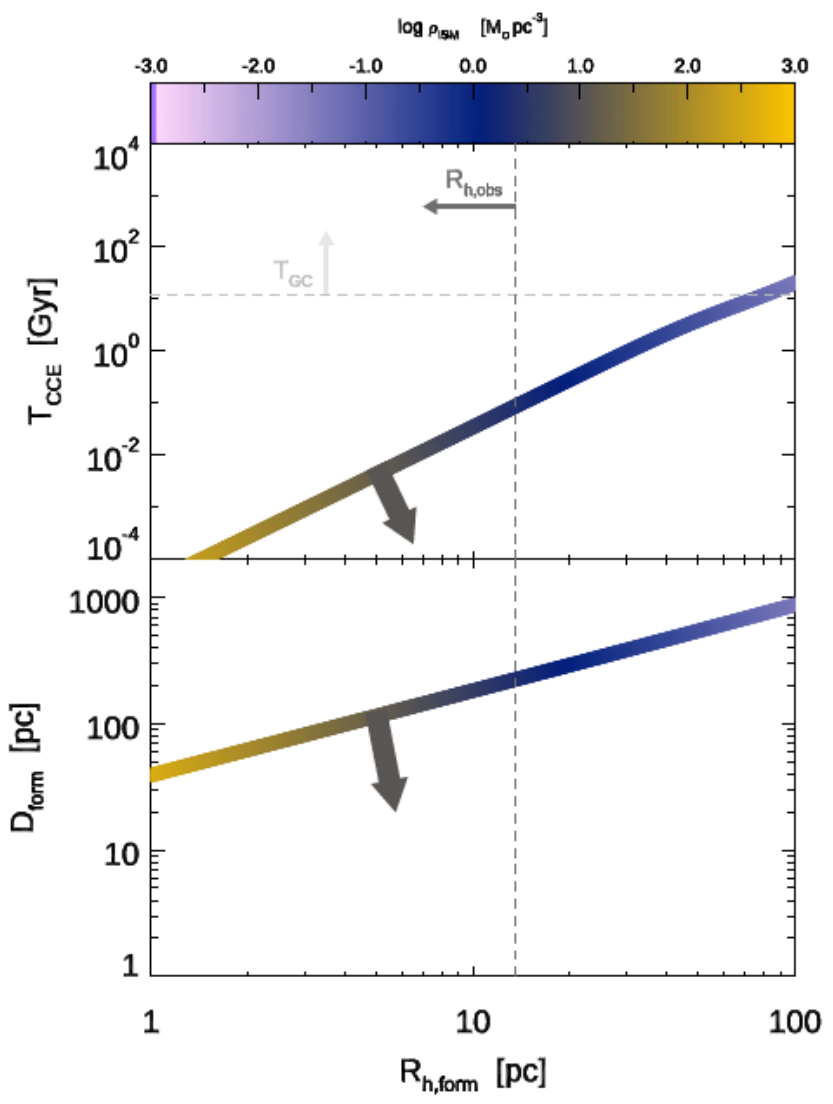

Figure B1. Bottom: limiting galactocentric formation distance in Pegasus for a GC forming in pressure equilibrium in an ISM with average density of $\rho_{\text {ISM }}$ (colour bar), as a function of the GC half-mass radius after formation. For a uniform galactic gas density distribution, the necessary pressures to form a GC like the one observed are not expected beyond $200 \mathrm{pc}$ in the dwarf galaxy - even for these conservative assumptions. Top: destruction timescale due to the gas tidal field as a function of the predicted GC formation size at the corresponding galaxy location. The predicted destruction timescales for GCs with correct sizes are shorter than the GC age implying that the GC should have likely migrated out of this environment on timescales of $\lesssim$ $100 \mathrm{Myr}$.

To form a GC with a size more typical of GCs in most galaxies ( $R_{\mathrm{h} \text {, form }} \sim 4 \mathrm{pc}$ ), the needed gas densities would require it to have been born even closer to the centre $\left(D_{\text {form }} \lesssim 100 \mathrm{pc}\right)$. Application of these physically motivated limiting values to this scenario suggests the required gas densities for such a massive GC would have likely been near the barycentre of its low-mass dwarf galaxy host.

The high pressures in the gas disc necessary to form such bound clusters, also pose a destructive risk to them (Kruijssen 2015). We can follow this study and ask on what time-scale the same highdensity gas peaks which helped the GC formed, would tidally shock and unbind the GC. The time-scale for destruction of a GC in a clumpy ISM of average density $\rho_{\text {ISM }}$ is expressed in Kruijssen (2015) as:

$T_{\mathrm{CCE}}=176 \mathrm{Myr}\left(\frac{M_{\mathrm{GC}}}{10^{5}}\right)\left(\frac{\rho_{\mathrm{gas}}}{\mathrm{M}_{\odot} \mathrm{pc}^{-3}}\right)^{-3 / 2}\left(\frac{f_{\Sigma}}{4}\right)^{-1} \phi_{\mathrm{ad}}^{-1}$

where $M_{\mathrm{GC}}$ is the mass of the GC and $\phi_{\mathrm{ad}}$ an adiabatic correction which is negligible for GCs of the mass of ours given our pressure equilibrium formation scenario. The GMC overdensity factor, $f_{\Sigma}$ is itself a function of gas density, and can be computed selfconsistently for our arbitrary choice of $\rho_{\text {gas }}$.
The coloured band in the top panel of Fig. B1 shows this GC survival time-scale as a function of the GC formation size, and an arbitrary choice of average gas density $\rho_{\text {gas }}$. The predicted timescales for survival of a GC forming in the inner region of our model galaxy are of order $\sim 10^{7-8} \mathrm{yr}-$ far less than the age of the GC.

This tension between the short hydrodynamical destruction times and the old GC ages was argued by Kruijssen (2015) to be one reason why GCs may need to migrate out of their high-density birth environments in order to survive until present. The simplified exercises in this paper which suggest a possible central formation, ejection and then inspiral of the GC in Pegasus, would be in qualitative agreement with this theoretical scenario.

How robust are some of the assumptions in this simplified picture? One could easily imagine that the molecular gas distribution at high redshifts had an extremely clumpy morphology, in which case local overdensities at any galactocentric distance might be possible (e.g. due to accretion in the outer disc of a galaxy). An overdensity of gas outside the central regions of the host is not captured in our above exercise, where we have considered the conservative limiting case of constant gas density.

Having the GC form from a massive gas cloud outside the central regions of its host galaxy is certainly possible - however in the case of Pegasus the large mass fraction of the GC relative to the galaxy might make it difficult for the natal GMC to be anything other than the centre of the gravitational potential in the galaxy.

The large gas fractions and SFRs accompanying high-redshift GC formation can result in a significant fraction of a galaxy's total star formation (upwards of 30 per cent; Kruijssen 2012; Larsen et al. 2012) occurring in bound clusters. Given the SFH of Pegasus, the initial cluster mass could have approached 50 per cent of the total mass in stars in the galaxy at those redshifts.

For the gas mass in the outer disc responsible for this event to not be the centre of mass of the system (e.g. for the GC to form outside of the centre of mass of the host galaxy) one requires $M_{\mathrm{gal}}$ enc $>$ $M_{\mathrm{GC}}$. Combining this requirement with equation (B2), we can write two inequalities to help assess this alternate scenario - namely under what conditions a massive GMC could be located outside of the galaxy centre (and not dominate the potential). The conditions require that the GMC is located beyond a distance and/or that the gas mass of the galaxy interior to that distance exceeds:

$$
\begin{aligned}
& D_{\text {form }}>\left(\frac{3 T_{\mathrm{CCE}} 10^{5} \rho^{1 / 2}}{4 \pi 1.76 \times 10^{8} \epsilon_{\mathrm{GMC}}}\right)^{1 / 3} \\
& M_{\text {gal, gas }}>\frac{T_{\mathrm{CCE}} 10^{5} \rho^{3 / 2}}{1.76 \times 10^{8} \epsilon_{\mathrm{GMC}}}
\end{aligned}
$$

Here, $\epsilon_{\mathrm{GMC}}$ is the gas-to-star conversion efficiency in the GMC which maps the GC mass in equation (B2) into a GMC mass.

In Fig. B2, we show curves of arbitrary gas density which span this galactocentric distance and galaxy gas mass parameter space for a particular choice of $\epsilon_{\mathrm{GMC}}$. The stellar mass of Pegasus at present day and the observed maximum SFR from Aparicio, Gallart \& Bertelli (1997) place constraints $\left(M_{*, \text { gal }} / \epsilon \lesssim M_{\text {gas, lim }} \lesssim \mathrm{SFR}_{\max } / \epsilon\right)$ on the allowable gas mass at fixed SFE, and these are indicated by the black solid lines. For reference, SFEs of $\epsilon_{\mathrm{GMC}}=0.01$ and 0.03 , typical in observations of molecular star-forming regions are shown as the dotted black lines.

Fig. B2 indicates that if the GC were to form in a massive GMC which was outside the centre of the galaxy, this would be possible out to distances of $D_{\text {form }} \lesssim 500 \mathrm{pc}$ without the GMC dominating the gravitational potential. Beyond this galactocentric distance, the SFE 




Figure B2. Required total galaxy gas mass as a function of GC formation distance such that the natal GMC does not become the galaxy centre of mass. Light coloured lines show these values for different GMC SFE $\epsilon_{\mathrm{GMC}}$. The black lines show the permitted gas mass values for Pegasus at that SFE, given the observed SFH and stellar mass constraints of Pegasus. Dashed grey lines show the present day stellar and gas mass, and virial mass of Pegasus. Dotted black lines indicate typical reference efficiencies seen observationally of $0.01 \leq \epsilon_{\mathrm{GMC}} \leq 0.03$ which bound constraints of $350 \lesssim D_{\text {form }} \lesssim 520 \mathrm{pc}$ and $8.2 \lesssim M_{\text {gal, gas }} \lesssim 8.7$. To have the GC form beyond this range would require lower GMC SFEs, but with galaxy gas masses in tension with the likely Pegasus virial mass at $z=2-6$ when the GC formed. would need to be lower than $\epsilon_{\mathrm{GMC}}=0.01$, but as a consequence the gas mass in the galaxy would need to increase - potentially approaching and in conflict with the virial mass of Pegasus at $z=2-6$ when the GC would have formed (Bermejo-Climent et al. 2018).

In summary, while we cannot fully know the geometry and physics of cluster formation at high redshift, some physically motivated limits suggest that this may have had to occur within the inner $\sim 500$ pc of Pegasus. Regardless of whether the massive GMC which formed the GC was more central than this, the GC likely would have needed to migrate out of the densest concentration of this gas on time-scales of $\sim 10-100 \mathrm{Myr}$. The smallest formation distance among our successful orbital inspiral trials is $D_{\text {form, }}=$ $712 \mathrm{pc}$, consistent with a scenario of migration away from a natal molecular gas environment to lower density regions of the host galaxy.

This paper has been typeset from a TEX/LTEX file prepared by the author. 